\title{
Background filtrations and
}

\section{canonical loss processes for top- down models of portfolio credit risk}

\section{Journal Article}

Author(s):

Ehlers, Philippe; Schoenbucher, Philipp J.

Publication date:

2009

Permanent link:

https://doi.org/10.3929/ethz-b-000117945

Rights / license:

In Copyright - Non-Commercial Use Permitted

Originally published in:

Finance and Stochastics 13(1), https://doi.org/10.1007/s00780-008-0080-x 


\title{
Background filtrations and canonical loss processes for top-down models of portfolio credit risk
}

\author{
Philippe Ehlers • Philipp J. Schönbucher
}

Received: 4 March 2007 / Accepted: 14 May 2008 / Published online: 18 November 2008

(C) Springer-Verlag 2008

\begin{abstract}
In single-obligor default risk modeling, using a background filtration in conjunction with a suitable embedding hypothesis (generally known as $\mathbb{H}$-hypothesis or immersion property) has proven a very successful tool to separate the actual default event from the model for the default arrival intensity. In this paper we analyze the conditions under which this approach can be extended to the situation of a portfolio of several obligors, with a particular focus on the so-called top-down approach. We introduce the natural $\mathbb{H}$-hypothesis of this setup (the successive $\mathbb{H}$-hypothesis) and show that it is equivalent to a seemingly weaker one-step $\mathbb{H}$-hypothesis. Furthermore, we provide a canonical construction of a loss process in this setup and provide closedform solutions for some generic pricing problems.
\end{abstract}

Keywords Credit risk · Default correlation · Point processes · Generalized Cox processes $\cdot$ Hypothesis $\mathbb{H}$

\section{JEL Classification G13}

Mathematics Subject Classification (2000) $60 \mathrm{G} 35 \cdot 91 \mathrm{~B} 28 \cdot 91 \mathrm{~B} 30$

\footnotetext{
Financial support by the National Centre of Competence in Research "Financial Valuation and Risk Management" (NCCR FINRISK) is gratefully acknowledged. NCCR FINRISK is a research program supported by the Swiss National Science Foundation (SNSF).

The authors would like to thank Monique Jeanblanc and two anonymous referees for their helpful comments and suggestions. Parts of this paper were presented at RiskDay 2006, Zurich. All remaining errors are our own. Comments and suggestions are very welcome.
}

P. Ehlers $(\varangle) \cdot$ P.J. Schönbucher

ETH Zurich, D-MATH, Rämistr. 101, 8092 Zurich, Switzerland

e-mail: Ehlers@math.ethz.ch

url: http://www.math.ethz.ch/ ehlers

P.J. Schönbucher

e-mail: P@Schonbucher.de

url: http://www.schonbucher.de 


\section{Introduction}

In single-obligor default risk modeling, using a background filtration in conjunction with an embedding hypothesis ( $\mathbb{H}$-hypothesis) has proven a very successful tool to separate the actual default event from the model for the default arrival intensity (see e.g. [19], or [11]). In particular, this approach allows to express the prices of all credit sensitive securities in terms of the default intensity alone, without reference to the actual default arrival process. This approach has become the de facto standard modeling approach for single-obligor intensity models.

For portfolio credit risk, there are two alternative approaches to set up intensitybased models. In the top-down approach, only the aggregate loss process of a given credit portfolio is modeled and the individual obligors are not referenced or identified. In the bottom-up approach, on the other hand, the default events of every individual obligor are modeled.

Top-down models have advantages in certain situations when the individual obligor in the portfolio "does not matter much": either-as in the case of the standard credit index reference portfolios ${ }^{1}$ —in cases when there is a liquid and important market for CDOs on the portfolio whose importance dwarfs the individual obligors, or-as in the case of retail portfolios or portfolios of small and medium-sized enterprises-in cases where the individual obligor is very small compared to the entire reference portfolio. In these cases, the simplification of the problem that is incurred in the top-down approach is outweighed by the gain in flexibility and tractability.

The strong growth of the markets for credit derivatives on standard index reference portfolios has increased the interest in the top-down credit models. Recent papers include [22-24] or [10], who use a forward-modeling approach similar to [15], and also $[12,14]$ or $[13]$, who do not use a forward-modeling approach but directly model the intensity of the portfolio's cumulative loss process. In the context of the theme of this paper it is particularly interesting that Sidenius et al. [23, 24] explicitly build their model around a setup with a background filtration, albeit without considering the details of the corresponding $\mathbb{H}$-hypothesis and its implications. Later on, we comment on the approach taken in $[23,24]$ in more detail.

Even more than in the single-obligor case, it is of great advantage for portfolio credit risk models if one can simplify the pricing problem to a problem which does not reference the loss process itself any more through an extension of the background filtration/ $/ \mathbb{H}$-embedding approach. We only mention the aspects of the numerical implementation of these models: Monte Carlo based methods will converge significantly faster if it is not necessary to simulate discrete events with extremely low probabilities (like the occurrence of large losses), and in Markov diffusion setups, the absence of jumps (i.e., the absence of the loss process) allows the use of standard solvers for the associated partial differential equations. For some pricing problems, even closedform solutions may become available.

In this paper we investigate possible extensions of the modeling approach with background filtrations and an embedding via an $\mathbb{H}$-hypothesis to the problem of port-

\footnotetext{
${ }^{1}$ The most liquid standard credit index portfolios are the iTraxx portfolio for European obligors and the CDX.NA portfolio of North-American obligors.
} 
folio credit risk modeling, with a specific focus on the top-down approach. It is well known that the implications of hypothesis $\mathbb{H}$ on the modeling of the credit risk of multiple obligors are stronger than the implications for single-obligor default models. For example (as observed by Kusuoka [18]) the most common setup for bottom-up models precludes the possibility of default contagion, i.e., the empirically observed phenomenon that at the default of an obligor, the default intensities of the other obligors jump up. We show in this paper that-as opposed to the bottom-up approach-in a top-down approach default contagion is compatible with the background filtration modeling approach. This is because the generalization of the $\mathbb{H}$-hypothesis proposed here is different from the one usually used in typical bottom-up setups like [5]: In a top-down approach we can exploit the fact that the event arrival times are ordered, while in a bottom-up approach one cannot make an a priori statement about the order in which the obligors will default. If one wanted to impose a similar "conditional Markov chain" structure (see Assumption 5.4) on a bottom-up model with $N$ obligors, the state space would be very large $\left(\{0,1\}^{N}\right)$ with many possible transitions, while in the top-down approach the state space is $\{0, \ldots, N\}$ with only one possible transition from each state. The only case where both approaches coincide is when the underlying portfolio is composed of $N$ exchangeable obligors (which remain exchangeable at all times).

The rest of the paper is structured as follows:

In Sect. 2, the mathematical framework of this paper is set up and we give a further illustration of the advantages of an $\mathbb{H}$-based pricing approach. Next, we introduce two different $\mathbb{H}$-type embedding assumptions: a successive $\mathbb{H}$-property and a (seemingly weaker) one-step $\mathbb{H}$-property, and show that in the setup of top-down portfolio credit risk modeling, the two assumptions actually are equivalent. This and related results on $\mathbb{H}$-embedding can be found in Sect. 3 .

In Sect. 4, we construct a canonical loss process such that the successive $\mathbb{H}$ property holds. This can be regarded as the portfolio analog of the well-known construction of default times with exponential random variables in the single-obligor case. Furthermore, we are also able to give some pricing formulae for defaultable claims which do not explicitly reference the loss process any more. As a complement to the canonical construction, we show in Sect. 4.2 that (under weak regularity conditions) the assumption of the $\mathbb{H}$-property is actually equivalent to the existence of a canonically constructed loss process so that the canonical construction indeed carries its name with justification.

One important special case (including e.g. the Sidenius et al. [23, 24] model) in which the complete conditional transition probabilities can be computed in closed form is the conditional Markov model which is treated in Sect. 5. In this situation the canonical loss process is a Markov chain when conditioned on the background filtration.

\section{Setup and conditional loss probabilities}

In pricing applications, the probability measure $\mathbf{P}$ should be viewed as spot martingale measure. For a vector $\underline{x}:=\left(x_{1}, \ldots, x_{N}\right)^{\prime} \in \mathbb{R}_{+}^{N}$, we denote by $\underline{x}^{k} \in \mathbb{R}^{k}$ the 
sub-vector of its first $k$ components, i.e., $\underline{x}^{k}:=\left(x_{1}, \ldots, x_{k}\right)^{\prime}$. Comparisons between vectors are understood componentwise.

\section{Assumption 2.1}

(i) The model is set up in a probability space $(\Omega, \mathcal{F}, \mathbf{P})$, which is equipped with an initial filtration/background filtration $\mathbb{F}^{0}=\left(\mathcal{F}_{t}^{0}\right)_{t \geq 0}$ satisfying the usual conditions (completeness and right-continuity).

(ii) On $(\Omega, \mathcal{F}, \mathbf{P})$, there is an increasing sequence of $N$ random times $0<\tau_{1}<\cdots<\tau_{N}<\infty$.

Our main object of investigation is a given portfolio of $N$ defaultable obligors. The random times $\tau_{1}<\cdots<\tau_{N}$ denote the ordered times of defaults in the portfolio, i.e., $\tau_{n}$ is the time of the $n$th default, and not necessarily the time of default of the $n$th obligor. For convenience we set $\tau_{0}:=0$. The loss process $L$ associated with the $\tau_{n}$ counts the number of defaults in the reference portfolio. Normalizing all losses in default to one, it is defined as

$$
L_{t}:=\sum_{n=1}^{N} \mathbf{1}_{\left\{\tau_{n} \leq t\right\}} .
$$

\subsection{Motivation of the pricing approach}

Synthetic CDOs are the most important ${ }^{2}$ portfolio credit derivatives on any given reference portfolio. In recent years, the markets for synthetic CDOs have gained significant liquidity and rather than being viewed as assets that have to be priced, index CDOs are now viewed as assets whose market prices are to be fitted by any model. CDOs also frequently serve as underlying assets for more exotic portfolio credit derivatives. Examples of such exotic portfolio credit derivatives are options on portfolio CDS, options on CDOs, forward-starting CDOs and in particular leveraged super-senior tranches (which are barrier options on CDOs).

Introducing CDOs as underlying assets to an exotic portfolio credit derivatives poses new challenges. Many classical models for portfolio credit risk (in particular the copula models) only model default arrivals. But in order to determine the exotic credit derivative's payoff, we need to value the (underlying) CDOs at the payoff date $t>0$, and for this it is necessary to have knowledge of the loss distribution at time $t$, i.e.,

$$
\left\{P_{n}(t, T):=\mathbf{P}\left[L_{T}=n \mid \mathcal{F}_{t}\right] \mid n=0, \ldots, N ; T>t\right\} .
$$

It is well known that knowledge of the loss distribution is necessary and sufficient to determine the prices of all synthetic CDOs at time $t$, i.e., to price CDOs for all attachment points and maturity dates.

As an example of a generic exotic portfolio credit derivative, we consider a contingent claim with maturity $t>0$ and payoff $X$ which can be written as a function $X=G\left(L_{t}, P_{n}(t, T), Z\right)$ of:

\footnotetext{
${ }^{2}$ We regard portfolio CDS as synthetic CDOs with attachment point $0 \%$ and detachment point $100 \%$.
} 
(i) losses $L_{t}$ up to time $t$

(ii) one representative component $P_{n}(t, T)$ of the loss distribution

(iii) other (not directly default-related) market variables $Z$ such as interest rates or exchange rates.

To shorten notation, we simplified the dependence on CDO prices from a dependence on the whole loss distribution $\left\{P_{n}(t, T) \mid n=0, \ldots, N ; T>t\right\}$ to a dependence on just one $P_{n}(t, T)$ alone. The argument would remain valid for any number of future loss probabilities $P_{n_{k}}\left(t, T_{k}\right), k=1, \ldots, K$, in the payoff function.

Let us consider two filtrations: a market filtration $\mathbb{F}=\left(\mathcal{F}_{t}\right)_{t \geq 0}$, and a background filtration $\mathbb{F}^{0}=\left(\mathcal{F}_{t}^{0}\right)_{t \geq 0}$, with the inclusion $\mathbb{F}^{0} \varsubsetneqq \mathbb{F}$ so that the background filtration contains less information than the market filtration. In particular, the loss process $L$ and the default times $\tau_{n}, n>0$, are $\mathbb{F}$-adapted, but not $\mathbb{F}^{0}$-adapted. The variable $Z$, on the other hand, is in the background information, i.e., $\mathcal{F}_{t}^{0}$-measurable.

We can now rewrite the payoff function $G(\cdot)$ as

$$
\begin{aligned}
X=G\left(L_{t}, P_{n}(t, T), Z\right) & =\sum_{m=0}^{N} \mathbf{1}_{\left\{L_{t}=m\right\}} G\left(m, P_{n}(t, T), Z\right) \\
& =\sum_{m=0}^{N} \mathbf{1}_{\left\{L_{t}=m\right\}} G\left(m, \mathbf{1}_{\left\{L_{t}=m\right\}} P_{n}(t, T), Z\right) .
\end{aligned}
$$

As we show in detail later on (Corollary 5.7 to Lemma 5.6 and (5.4)), under suitable assumptions, we have that

$$
\mathbf{1}_{\left\{L_{t}=k\right\}} P_{n}(t, T)=\mathbf{1}_{\left\{L_{t}=k\right\}} \widetilde{P}_{n, k}(t, T),
$$

where the variables $\widetilde{P}_{n, k}(t, T)$ are only $\mathbb{F}^{0}$-adapted, i.e., they do not directly reference the loss process any more. The payoff decomposition becomes

$$
X=\sum_{m=0}^{N} \mathbf{1}_{\left\{L_{t}=m\right\}} G\left(m, \mathbf{1}_{\left\{L_{t}=m\right\}} \widetilde{P}_{n, m}(t, T), Z\right)=\sum_{m=0}^{N} \mathbf{1}_{\left\{L_{t}=m\right\}} G\left(m, \widetilde{P}_{n, m}(t, T), Z\right),
$$

and the problem of pricing the generic contingent claim reduces to the evaluation of

$$
\begin{aligned}
\mathbf{E}[X] & =\sum_{m=0}^{N} \mathbf{E}\left[\mathbf{1}_{\left\{L_{t}=m\right\}} G\left(m, \widetilde{P}_{n, m}(t, T), Z\right)\right] \\
& =\sum_{m=0}^{N} \mathbf{E}\left[\mathbf{E}\left[\mathbf{1}_{\left\{L_{t}=m\right\}} G\left(m, \widetilde{P}_{n, m}(t, T), Z\right) \mid \mathcal{F}_{t}^{0}\right]\right] \\
& =\sum_{m=0}^{N} \mathbf{E}\left[\mathbf{P}\left[L_{t}=m \mid F_{t}^{0}\right] G\left(m, \widetilde{P}_{n, m}(t, T), Z\right)\right] .
\end{aligned}
$$

In the last equation all variables are $\mathcal{F}_{t}^{0}$-measurable; thus all reference to the loss process has been removed and the pricing problem has been reduced to a problem under the background filtration $\mathbb{F}^{0}$. 
A numerical scheme to evaluate (2.2) using Monte Carlo simulation would involve the following steps for each scenario of the background variables:

(i) evaluate $\mathbf{P}\left[L_{t}=m \mid \mathcal{F}_{t}^{0}\right]$

(ii) evaluate $\widetilde{P}_{n, m}(t, T)$, and

(iii) repeat and average the results.

(Of course, based upon the concrete specification of the processes, one may find more efficient algorithms.)

Step (i) requires integrating a set of integrals (given in Lemma 5.6) which can be done quite efficiently. It is here where the numerical advantage of the background approach lies. In the market filtration, one would have to average over a very large number of simulated paths for the loss process $L$ to reach the same "weights" that are reached here with one integration: Large losses typically have very low probabilities $\left(\approx 10^{-3}\right)$, yet the payoffs in these states are usually highly relevant to the credit derivative's value. Thus, a very large number of simulations is required to accurately capture the probabilities of these states of nature.

Step (ii) is probably numerically the most expensive step. It can be achieved by either using projection methods similar to the Longstaff and Schwartz [20] leastsquares approach, by running a second "simulation within the simulation," or by exploiting possible additional structure in the model specification (e.g. Markovianity with a low-dimensional state space).

The numerical effort for the evaluation of the future price of the underlying CDO in step (ii) is not specific to the background filtration approach. It will pose the same numerical problems in almost all models; in particular it will still be present (and even more important, because it has to be performed in many more scenarios) if the background approach is not used.

The following questions were left open in the argument above and will be answered in this paper:

- What assumptions are necessary (and/or sufficient) for (2.1) to hold?

- Under these assumptions, can we give closed-form expressions for $\widetilde{P}_{n, k}(t, T)$ ?

- Under these assumptions, can we give closed-form expressions for $\mathbf{P}\left[L_{t}=m \mid F_{t}^{0}\right]$ ?

\subsection{Filtrations}

Filtrations are abbreviated with math blackboard letters and the $\sigma$-algebras that make up the filtration with math calligraphic letters (e.g. $\mathbb{G}$ stands for $\left.\left(\mathcal{G}_{t}\right)_{t \geq 0}\right) \cdot \mathcal{M}^{2}(\mathbb{G})$ denotes the space of square-integrable $\mathbb{G}$-martingales, and all processes are assumed to be càdlàg (unless explicitly specified otherwise).

\section{Definition 2.2 (Filtrations)}

(i) For each $n, \mathbb{F}^{n}$ is the smallest filtration which contains $\mathbb{F}^{0}$, satisfies the usual hypotheses, and makes $\tau_{1}, \ldots, \tau_{n}$ stopping times. In other words,

$$
\mathcal{F}_{t}^{n}=\bigcap_{\epsilon>0}\left(\mathcal{F}_{t+\epsilon}^{0} \vee \mathcal{T}_{t+\epsilon}^{1} \vee \cdots \vee \mathcal{T}_{t+\epsilon}^{n}\right)
$$

where $\mathbb{T}^{n}$ is given by $\mathcal{T}_{t}^{n}=\sigma\left(\left\{\left\{\tau_{n}>u\right\} ; u \leq t\right\}\right)$. 
(ii) The market filtration is the filtration $\mathbb{F}:=\mathbb{F}^{N}$ containing information about all default times in the portfolio.

Clearly, $L$ is $\mathbb{F}^{N}$-adapted and $\mathbb{F}^{0} \subseteq \mathbb{F}^{1} \subseteq \cdots \subseteq \mathbb{F}^{N} . \mathbb{F}^{0}$ can be thought of as the flow of default-free market information to which all assets that are insensitive to credit portfolio losses are adapted, and $\mathbb{F}=\mathbb{F}^{N}$ is the full market filtration, including information about all defaults.

In order to avoid trivialities, we assume that $\mathbb{F}^{n}$ does not contain "too much" information about the next loss time $\tau_{n+1}$ :

Assumption 2.3 $\mathbf{P}\left[\tau_{n+1}>t \mid \mathcal{F}_{t}^{n}\right]>0$ a.s. for every $n=0, \ldots, N-1$ and $t \geq 0$.

Then, since also $\tau_{n+1}<\infty$ a.s., $\tau_{n+1}$ is in particular not an $\mathbb{F}^{n}$-stopping time.

Remark $2.4 \mathbb{F}^{n}$ is not equal to $\mathbb{G}^{n}:=\mathbb{F}^{0} \vee \mathbb{T}^{1} \vee \cdots \vee \mathbb{T}^{n}$, the minimal enlargement of $\mathbb{F}^{0}$ which makes $\tau_{1}, \ldots, \tau_{n}$ stopping times: $\mathbb{G}^{n}$ is in general not right-continuous (see e.g. [21], p. 370), while $\mathbb{F}^{n}$ is. We chose to use $\mathbb{F}^{n}$ as we find it more convenient to work with the minimal enlargements that do satisfy the usual conditions. Nevertheless, almost all results obtained in this paper remain valid if $\mathbb{F}^{n}$ is replaced with $\mathbb{G}^{n}$ for all $n$.

Also note that $\mathbb{F}^{n+1}$ can be characterized equivalently in recursive fashion as the smallest filtration which contains $\mathbb{F}^{n}$, makes $\tau_{n+1}$ a stopping time, and satisfies the usual conditions, i.e.,

$$
\mathcal{F}_{t}^{n+1}=\bigcap_{\epsilon>0}\left(\mathcal{F}_{t+\epsilon}^{0} \vee \mathcal{T}_{t+\epsilon}^{1} \vee \cdots \vee \mathcal{T}_{t+\epsilon}^{n+1}\right)=\bigcap_{\epsilon>0}\left(\mathcal{F}_{t+\epsilon}^{n} \vee \mathcal{T}_{t+\epsilon}^{n+1}\right) .
$$

An explicit generator $\pi_{t}^{n}$ of the $\sigma$-field $\mathcal{F}_{t}^{0} \vee \mathcal{T}_{t}^{1} \vee \cdots \vee \mathcal{T}_{t}^{n}$ is

$$
\pi_{t}^{n}:=\left\{F^{0} \cap\left\{\underline{\tau}^{n}>\underline{u}^{n}\right\} ; F^{0} \in \mathcal{F}_{t}^{0}, \underline{u} \in[0, t]^{N}\right\}
$$

and the $\sigma$-field $\mathcal{F}_{t}^{n-1} \vee \mathcal{T}_{t}^{n}$ is generated by the $\pi$-system

$$
\tilde{\pi}_{t}^{n}:=\left\{F \cap\left\{\underline{\tau}^{n}>\underline{u}^{n}\right\} ; F \in \mathcal{F}_{t}^{n-1}, \underline{u} \in[0, t]^{N}\right\} .
$$

\section{The successive $\mathbb{H}$-property}

Definition 3.1 (i) below is the standard definition of the martingale preserving property/embedding property ( $\mathbb{H}$-hypothesis) in the case when the enlargement of the filtration is done in one step, i.e. if only two filtrations are involved. In the topdown credit loss setup, on the other hand, a succession of $N$ filtration enlargements $\mathbb{F}^{0} \subseteq \mathbb{F}^{1} \subseteq \cdots \subseteq \mathbb{F}^{N}$ exists. We define two extensions of the $\mathbb{H}$-hypothesis: 
Definition 3.1 (H-hypothesis)

(i) Let $\mathbb{F}$ be a subfiltration of $\mathbb{G}$. The enlargement $\mathbb{G} \supseteq \mathbb{F}$ satisfies the $\mathbb{H}$-property and we write $\mathbb{F} \stackrel{\mathbb{H}}{\longrightarrow} \mathbb{G}$ iff

$$
\mathcal{M}^{2}(\mathbb{F}) \subseteq \mathcal{M}^{2}(\mathbb{G})
$$

(ii) $\mathbb{F}^{0} \subseteq \mathbb{F}^{1} \subseteq \cdots \subseteq \mathbb{F}^{N}$ satisfies the successive $\mathbb{H}$-property iff

$$
\mathbb{F}^{n} \stackrel{\mathbb{H}}{\longrightarrow} \mathbb{F}^{n+1} \quad \text { for every } n=0, \ldots, N-1 .
$$

(iii) $\mathbb{F}^{0} \subseteq \mathbb{F}^{1} \subseteq \cdots \subseteq \mathbb{F}^{N}$ satisfies the one-step $\mathbb{H}$-property iff

$$
\mathbb{F}^{0} \stackrel{\mathbb{H}}{\longrightarrow} \mathbb{F}^{N}
$$

Remark 3.2 In general, the successive $\mathbb{H}$-property $(*)$ is a stronger assumption than the one-step $\mathbb{H}$-property $(* *)$, i.e., in an arbitrary successive enlargement $\mathbb{G}^{I} \subseteq \mathbb{G}^{I I} \subseteq \mathbb{G}^{I I I}, \mathbb{G}^{I} \stackrel{\mathbb{H}}{\longrightarrow} \mathbb{G}^{I I I}$ does in general not imply $\mathbb{G}^{I I} \stackrel{\mathbb{H}}{\longrightarrow} \mathbb{G}^{I I I}$ although it does of course imply $\mathbb{G}^{I} \stackrel{\mathbb{H}}{\longrightarrow} \mathbb{G}^{I I}$.

As a simple counterexample take an enlargement $\mathbb{G}^{I I} \subseteq \mathbb{G}^{I I I}$ which does not satisfy the $\mathbb{H}$-hypothesis ${ }^{3}$ and let $\mathbb{G}^{I}$ be trivial. Then $\mathbb{G}^{I} \stackrel{\mathbb{H}}{\longrightarrow} \mathbb{G}^{I I I}$ holds, but of course by construction we do not have $\mathbb{G}^{I I} \stackrel{\mathbb{H}}{\longrightarrow} \mathbb{G}^{I I I}$.

The following theorem is the main result of this section. It states that in the case of a successive enlargement by an increasing sequence of stopping times, the one-step $\mathbb{H}$-property is actually already sufficient for the successive $\mathbb{H}$-property:

Theorem 3.3 The following assertions are equivalent in our enlargement setup:

(i) $\mathbb{F}^{0} \stackrel{\mathbb{H}}{\longrightarrow} \mathbb{F}^{N}$ : The one-step $\mathbb{H}$ property (**) holds.

(ii) $\mathbb{F}^{n} \stackrel{\mathbb{H}}{\longrightarrow} \mathbb{F}^{n+1}$ for every $n=0, \ldots, N-1$ : The successive $\mathbb{H}$-property $(*)$ holds.

(iii) $\mathbb{F}^{n} \stackrel{\mathbb{H}}{\longrightarrow} \mathbb{F}^{N}$ for every $n=0, \ldots, N-1$.

Proof See Sect. 3.1 below.

Remark 3.4 Theorem 3.3 can be extended in a straightforward manner to the case $N=\infty$. For an increasing sequence of random times $\tau_{n}, n \in \mathbb{N}$, let $\mathbb{F}^{\infty}$ be the minimal filtration satisfying the usual hypotheses, which contains $\mathbb{F}^{0}$ and makes all $\tau_{n}$ stopping times; in other words, $\mathcal{F}_{t}^{\infty}:=\bigcap_{\epsilon>0}\left(\mathcal{F}_{t+\epsilon}^{0} \vee \bigvee_{n=0}^{\infty} \mathcal{T}_{t+\epsilon}^{n}\right)$. Then the following assertions are equivalent:

(i') $\mathbb{F}^{0} \stackrel{\mathbb{H}}{\longrightarrow} \mathbb{F}^{\infty}$.

\footnotetext{
${ }^{3}$ E.g. let $\mathbb{G}^{I I}$ be the natural filtration of a Brownian motion $W$ on $[0,1]$, and let $\mathbb{G}^{I I I}$ be generated by adding information about its final value, i.e., $\mathcal{G}_{t}^{I I I}=\sigma\left(W_{1}\right) \vee \mathcal{G}_{t}^{I I}$ for all $t \leq 1$.
} 
(ii') $\mathbb{F}^{n} \stackrel{\mathbb{H}}{\longrightarrow} \mathbb{F}^{n+1}$ for all $n \in \mathbb{N}$.

(iii') $\mathbb{F}^{n} \stackrel{\mathbb{H}}{\longrightarrow} \mathbb{F}^{\infty}$ for all $n \in \mathbb{N}$.

Noting that $\mathcal{F}_{t}^{0} \vee \bigvee_{n=0}^{\infty} \mathcal{T}_{t}^{n}$ is generated by sets of the form $F^{0} \cap\left\{\underline{\tau}^{N}>\underline{u}^{N}\right\}$ with $F^{0} \in \mathcal{F}_{t}^{0}, \underline{u}^{N} \in[0, t]^{N}$ and $N \in \mathbb{N}$, the proof is analogous to that of Theorem 3.3.

Theorem 3.3 states that if (i) adding the full running loss information $\bigvee_{n=1}^{N} \mathbb{T}^{n}$ to the default-free market information $\mathbb{F}^{0}$ "at once" preserves the martingale property, then (ii) "nothing can go wrong" any more in the intermediate steps; the successive $\mathbb{H}$-assumption already holds.

It is hard to argue directly why the successive $\mathbb{H}$-assumption $(*)$ would hold in real markets. This would require several arguments for each of the successive default times $\tau_{n}$ and their corresponding enlargements of filtrations $\mathbb{F}^{n} \stackrel{\mathbb{H}}{\longrightarrow} \mathbb{F}^{n+1}$, moving from one hypothetical situation ("if we could only observe the first $n$ defaults ...") to another hypothetical situation ("if we could only observe the first $n+1$ defaults ..."). Both of these hypothetical situations are rather contrived. (Why do we only observe the first $n$ defaults? Why not more? Why not less? Why any defaults at all? Which $N-n$ obligors out of all $N$ obligors do we not observe-what are their names?) Thus, forming an opinion about the differences between them (i.e., validating $\mathbb{F}^{n} \stackrel{\mathbb{H}}{\longrightarrow} \mathbb{F}^{n+1}$ with an economic argument) seems almost impossible.

Fortunately, Theorem 3.3 relieves us from this task and tells us that it is sufficient to motivate $(* *)$, i.e., $\mathbb{F}^{0} \stackrel{\mathbb{H}}{\longrightarrow} \mathbb{F}^{N}$. This is much easier. First, there is only one hypothesis to support, and not $N$. Second, that hypothesis is about the difference between the situation when we observe no defaults (i.e., $\mathbb{F}^{0}$ ), and the situation when we observe all defaults (i.e., $\mathbb{F}^{N}$ ). The situation $\mathbb{F}^{N}$ is the market filtration; it is the information that is available in reality, not a hypothetical situation. And information $\mathbb{F}^{0}$, i.e., not observing any defaults, is also a clearly defined situation that one can imagine without difficulty in a thought experiment.

Nevertheless, Theorem 3.3 does not do all the work for us. It does not in itself provide a "proof" of the one-step $\mathbb{H}$-hypothesis (**). The condition (**) is still an assumption which has to be justified from economic arguments outside the mathematical model.

Furthermore, it was noticed by Kusuoka [18] that the $\mathbb{H}$-hypothesis is in general not invariant under an equivalent change of probability measure. Hence, for derivatives pricing, assumption $\mathbb{F}^{0} \stackrel{\mathbb{H}}{\longrightarrow} \mathbb{F}^{N}$ has to be made directly under the/an equivalent martingale (pricing) measure. There are some interesting related results, e.g. it can be shown that if the defaultable/loss $\mathbb{F}^{N}$-market is arbitrage-free and the default-free/initial $\mathbb{F}^{0}$-market is complete, then $\mathbb{F}^{0} \stackrel{\mathbb{H}}{\longrightarrow} \mathbb{F}^{N}$ is satisfied under every $\mathbb{F}^{N}$-equivalent martingale measure (see [3], p. 150).

While the one-step $\mathbb{H}$-property $(* *)$ is much easier to justify, it is unfortunately less convenient to work with (otherwise we should not need Theorem 3.3). For concrete calculations, the successive $\mathbb{H}$-property has the advantage of allowing a reduction of the pricing problems to an iterative sequence of problems, where the $n$th problem only involves one stopping time $\tau_{n}$. 
Problems involving one stopping time under $\mathbb{H}$ have already been treated extensively in the literature. The main workhorse for such problems is the following wellknown lemma (see e.g. [4] or [11]) which summarizes the key properties of expectations under filtration enlargements satisfying $\mathbb{H}$ :

Lemma 3.5 The following statements are equivalent to $\mathbb{F} \stackrel{\mathbb{H}}{\longrightarrow} \mathbb{G}$.

(i) $\mathbf{E}\left[F \mid \mathcal{F}_{t}\right]=\mathbf{E}\left[F \mid \mathcal{G}_{t}\right]$ for every $F \in L^{2}\left(\mathcal{F}_{\infty}\right)$ for any $t$.

(ii) $\mathbf{E}\left[G \mid \mathcal{F}_{\infty}\right]=\mathbf{E}\left[G \mid \mathcal{F}_{t}\right]$ for every $G \in L^{2}\left(\mathcal{G}_{t}\right)$ for any $t$.

(iii) $\mathbf{E}\left[F G \mid \mathcal{F}_{t}\right]=\mathbf{E}\left[F \mid \mathcal{F}_{t}\right] \mathbf{E}\left[G \mid \mathcal{F}_{t}\right]$ for every $F \in L^{2}\left(\mathcal{F}_{\infty}\right)$ and $G \in L^{2}\left(\mathcal{G}_{t}\right)$ for any $t$, i.e., $\mathcal{F}_{\infty}$ and $\mathcal{G}_{t}$ are conditionally independent given $\mathcal{F}_{t}$ for any $t$.

Proof See the references in [11].

\subsection{Proof of Theorem 3.3}

The proof, apart from some technical details, is based on the monotonicity of the random times $\tau_{1}, \ldots, \tau_{N}$ and the explicit generator $\tilde{\pi}_{t}^{n}$ of $\mathcal{F}_{t}^{n-1} \vee \mathcal{T}_{t}^{n}$ as given in (2.3).

We need an auxiliary lemma concerning general enlargements (starting from filtrations which satisfy the usual hypotheses).

Lemma 3.6 Let $\mathbb{G}$ be any filtration and $\mathbb{G}^{+}$be the minimal right-continuous filtration containing $\mathbb{G}$.

(i) Every bounded $\mathbb{G}$-martingale with a càdlàg version is also a $\mathbb{G}^{+}$-martingale.

(ii) Let $\mathbb{F}$ satisfy the usual conditions and $\mathbb{F} \subseteq \mathbb{G}$. Then $\mathbb{F} \stackrel{\mathbb{H}}{\longrightarrow} \mathbb{G}$ implies $\mathbb{F} \stackrel{\mathbb{H}}{\longrightarrow} \mathbb{G}^{+}$.

Proof (i) can be found in [6], Chap. IV, Theorem 2, and (ii) follows directly from (i).

Proof of Theorem 3.3 It is clear that "(iii) $\Rightarrow$ (ii)" and "(ii) $\Rightarrow$ (i)". To show "(i) $\Rightarrow$ (iii)", we shall prove that $\mathbb{F}^{0} \stackrel{\mathbb{H}}{\longrightarrow} \cdots \stackrel{\mathbb{H}}{\longrightarrow} \mathbb{F}^{k-1} \stackrel{\mathbb{H}}{\longrightarrow} \mathbb{F}^{N}$ implies $\mathbb{F}^{k-1} \stackrel{\mathbb{H}}{\longrightarrow} \mathbb{F}^{k} \stackrel{\mathbb{H}}{\longrightarrow} \mathbb{F}^{N}$ for every $k=1, \ldots, N-1$. Then the claim will follow by induction in $k$.

So let $\mathbb{F}^{0} \stackrel{\mathbb{H}}{\longrightarrow} \cdots \stackrel{\mathbb{H}}{\longrightarrow} \mathbb{F}^{k-1} \stackrel{\mathbb{H}}{\longrightarrow} \mathbb{F}^{N}$ hold for some $0<k<N$. Since $\mathbb{F}^{k} \subseteq \mathbb{F}^{N}$, it remains to show $\mathbb{F}^{k} \stackrel{\mathbb{H}}{\longrightarrow} \mathbb{F}^{N}$. By virtue of Lemma 3.5 and Lemma 3.6 (ii), we must only prove

$$
\mathbf{E}\left[X^{k} \mid \mathcal{F}_{t}^{k} \vee \mathcal{T}_{t}^{k+1} \vee \cdots \vee \mathcal{T}_{t}^{N}\right]=\mathbf{E}\left[X^{k} \mid \mathcal{F}_{t}^{k}\right] \text { for all } X^{k} \in L^{2}\left(\mathcal{F}_{\infty}^{k}\right),
$$

and hence by Dynkin's lemma, it is sufficient to show that for any $\underline{u}^{N} \in[0, t]^{N}$ and $F^{k} \in \mathcal{F}_{t}^{k}$,

$$
\mathbf{E}\left[X^{k} \mathbf{1}_{\left\{\underline{\tau}^{N}>\underline{u}^{N}\right\}} \mathbf{1}_{F^{k}}\right]=\mathbf{E}\left[\mathbf{E}\left[X^{k} \mid \mathcal{F}_{t}^{k}\right] \mathbf{1}_{\left\{\underline{\tau}^{N}>\underline{u}^{N}\right\}} \mathbf{1}_{F^{k}}\right] .
$$

We first take $X^{k}$ of the form $X^{k}=X^{k-1} \mathbf{1}_{\left\{\tau_{k}>T\right\}}$ where $X^{k-1} \in L^{2}\left(\mathcal{F}_{\infty}^{k-1}\right)$ and $T \geq 0$. By assumption, it holds $\mathbf{E}\left[X^{k-1} \mid \mathcal{F}_{t}^{N}\right]=\mathbf{E}\left[X^{k-1} \mid \mathcal{F}_{t}^{k-1}\right]=\mathbf{E}\left[X^{k-1} \mid \mathcal{F}_{t}^{k}\right]$. Thus, if 
$T<t$, we have

$$
\begin{aligned}
\mathbf{E}\left[X^{k} \mathbf{1}_{\left\{\underline{\tau}^{N}>\underline{u}^{N}\right\}} \mathbf{1}_{F^{k}}\right] & =\mathbf{E}\left[\mathbf{E}\left[X^{k-1} \mid \mathcal{F}_{t}^{N}\right] \mathbf{1}_{\left\{\tau_{k}>T\right\}} \mathbf{1}_{\left\{\underline{\tau}^{N}>\underline{u}^{N}\right\}} \mathbf{1}_{F^{k}}\right] \\
& \stackrel{\mathbb{H}}{=} \mathbf{E}\left[\mathbf{E}\left[X^{k-1} \mid \mathcal{F}_{t}^{k}\right] \mathbf{1}_{\left\{\tau_{k}>T\right\}} \mathbf{1}_{\left\{\underline{\tau}^{N}>\underline{u}^{N}\right\}} \mathbf{1}_{F^{k}}\right] \\
& =\mathbf{E}\left[\mathbf{E}\left[X^{k-1} \mathbf{1}_{\left\{\tau_{k}>T\right\}} \mid \mathcal{F}_{t}^{k}\right] \mathbf{1}_{\left\{\underline{\tau}^{N}>\underline{u}^{N}\right\}} \mathbf{1}_{F^{k}}\right] \\
& =\mathbf{E}\left[\mathbf{E}\left[X^{k} \mid \mathcal{F}_{t}^{k}\right] \mathbf{1}_{\left\{\underline{\tau}^{N}>\underline{u}^{N}\right\}} \mathbf{1}_{F^{k}}\right] .
\end{aligned}
$$

On the other hand, if $T \geq t$, we notice that thanks to the monotonicity of $\tau_{1}, \ldots, \tau_{N}$ we have $\mathbf{1}_{\left\{\tau_{k}>T\right\}}=\mathbf{1}_{\left\{\tau_{k}>T\right\}} \mathbf{1}_{\left\{\tau_{k}>t\right\}}$ and $\mathbf{1}_{\left\{\tau_{k}>t\right\}} \mathbf{1}_{\left\{\underline{\tau}^{N}>\underline{u}^{N}\right\}}=\mathbf{1}_{\left\{\tau_{k}>t\right\}} \mathbf{1}_{\left\{\underline{\tau}^{k}>\underline{u}^{k}\right\}}$. Hence

$$
\begin{aligned}
\mathbf{E}\left[X^{k} \mathbf{1}_{\left\{\underline{\tau}^{N}>\underline{u}^{N}\right\}} \mathbf{1}_{F^{k}}\right] & =\mathbf{E}\left[X^{k-1} \mathbf{1}_{\left\{\tau_{k}>T\right\}} \mathbf{1}_{\left\{\tau_{k}>t\right\}} \mathbf{1}_{\left\{\underline{\tau}^{k}>\underline{u}^{k}\right\}} \mathbf{1}_{F^{k}}\right] \\
& =\mathbf{E}\left[\mathbf{E}\left[X^{k-1} \mathbf{1}_{\left\{\tau_{k}>T\right\}} \mid \mathcal{F}_{t}^{k}\right] \mathbf{1}_{\left\{\tau_{k}>t\right\}} \mathbf{1}_{\left\{\underline{\tau}^{k}>\underline{u}^{k}\right\}} \mathbf{1}_{F^{k}}\right] \\
& =\mathbf{E}\left[\mathbf{E}\left[X^{k-1} \mathbf{1}_{\left\{\tau_{k}>T\right\}} \mid \mathcal{F}_{t}^{k}\right] \mathbf{1}_{\left\{\tau_{k}>t\right\}} \mathbf{1}_{\left\{\underline{\tau}^{N}>\underline{u}^{N}\right\}} \mathbf{1}_{F^{k}}\right] \\
& =\mathbf{E}\left[\mathbf{E}\left[X^{k} \mid \mathcal{F}_{t}^{k}\right] \mathbf{1}_{\left\{\underline{\tau}^{N}>\underline{u}^{N}\right\}} \mathbf{1}_{F^{k}}\right] .
\end{aligned}
$$

Then the claim follows because $\left\{X^{k}=X^{k-1} \mathbf{1}_{\left\{\tau_{k}>T\right\}} \mid X^{k-1} \in L^{2}\left(\mathcal{F}_{\infty}^{k-1}\right), T \geq 0\right\}$ is dense in $L^{2}\left(\mathcal{F}_{\infty}^{k}\right)$.

\section{The canonical loss process}

The aim of this section is to provide a concrete situation in which the successive $\mathbb{H}$-property holds: the canonical construction of a loss process. Proposition 4.9 will show that this construction is indeed the stochastic representation of the successive $\mathbb{H}$-property: Up to regularity, assuming the successive $\mathbb{H}$-property $(*)$ is equivalent to assuming the construction below.

\subsection{The canonical construction}

As we want to construct random times $\tau_{1}, \ldots, \tau_{N}$ satisfying $(*)$ (they are not given a priori), we need some "construction material":

Assumption 4.1 On $(\Omega, \mathcal{F}, P)$ there are $N$ random variables $E_{1}, \ldots, E_{N}$ which are i.i.d. unit-exponentially distributed and independent of $\mathcal{F}_{\infty}^{0}$.

Definition 4.2 (Canonical construction) Let Assumption 4.1 hold.

(A) For each $n=0, \ldots, N-1$, successively

(n1) choose $\lambda^{n} \geq 0, \mathbb{F}^{n}$-adapted with $\int_{0}^{t} \lambda_{s}^{n} d s<\infty$ a.s. for all $t<\infty$ and $\int_{0}^{\infty} \lambda_{s}^{n} d s=\infty$ a.s.

(n2) define $\tau_{n+1}:=\inf \left\{t>\tau_{n} \mid \int_{\tau_{n}}^{t} \lambda_{s}^{n} d s \geq E_{n+1}\right\}$

(n3) let $\mathbb{F}^{n+1}$ be as in Definition 2.2. 
(B) Define the loss process $L_{t}:=\sum_{n=1}^{N} \mathbf{1}_{\left\{\tau_{n} \leq t\right\}}$ and let $\mathbb{F}^{N}$ be the market filtration

(C) We call $L$ the canonical loss process with respect to $\lambda^{n} ; A$ denotes the predictable $\mathbb{F}^{N}$-compensator of $L$.

For the one-obligor case $(N=1)$ this is the Cox process construction by Lando [19]. Here, $L$ can be interpreted as a generalization of the Cox process construction because $\lambda^{n}$ may vary across different values of $n$, and furthermore we allow $\lambda^{n}$ to depend on (a part of) the history of $L$, namely the "truncated" loss history $\sigma\left(\left\{L_{u} \wedge n, u \leq t\right\}\right)$. Nevertheless, as in [19], also here the "intensity" $\lambda_{t}^{n}$ is welldefined even after $\tau_{n}$.

Here are some elementary properties of the canonically constructed loss process.

Proposition 4.3 The canonical loss process has the following properties.

P1. $\tau_{0}<\tau_{1}<\cdots<\tau_{N}<\infty$ a.s.

P2. $\mathbf{P}\left[\tau_{n+1}>t \mid \mathcal{F}_{\infty}^{n}\right]=e^{-\int_{t \wedge \tau_{n}}^{t} \lambda_{s}^{n} d s}$.

P3. The successive $\mathbb{H}$-property $(*)$ holds.

P4. $A_{t}=\sum_{n=0}^{N-1} \int_{0}^{t} \mathbf{1}_{\left\{L_{s}=n\right\}} \lambda_{s}^{n} d s$.

P5. $\tau_{n+1}$ avoids the $\mathbb{F}^{n}$-stopping times, i.e., for every $\mathbb{F}^{n}$-stopping time $\Theta$, $\mathbf{P}\left[\tau_{n+1}=\Theta\right]=0$.

Proof See Appendix A.

Properties P1 and P2 ensure that Assumption 2.3 holds and by P4 the canonical loss process admits the $\mathbb{F}^{N}$-intensity

$$
\lambda_{t}:=\sum_{n=0}^{N-1} \mathbf{1}_{\left\{L_{t}=n\right\}} \lambda_{t}^{n} .
$$

This shows that $\tau_{n}$ is $\mathbb{F}^{N}$ - (and hence $\mathbb{F}^{n}$-) totally inaccessible for each $n$ and that contagion, usually referred to as the ability of the loss intensity to jump (up) at the occurrence of defaults, is a natural feature of the canonical model. The size of the jump of the loss intensity at the $n$th default is $\Delta \lambda_{\tau_{n}}=\lambda_{\tau_{n}}^{n}-\lambda_{\tau_{n}-}^{n-1}$ for all $n$.

Remark 4.4 Enlargements by (a sequence of) ordered random times were also studied in [16], Chap. 5.1. They let $\Psi^{1} \leq \Psi^{2}$ be nonincreasing $\mathbb{F}^{0}$-adapted processes and define

$$
\tau_{n}:=\inf \left\{t \geq 0 \mid e^{-\Psi_{t}^{n}} \geq E\right\}, \quad n=1,2,
$$

where $E$ is one unit exponential random variable independent of $\mathcal{F}_{\infty}^{0}$. This construction is evidently different from ours. In particular, in their setup $\mathcal{F}_{\infty}^{2}$ is generated by $\mathcal{F}_{\infty}^{0}$ and $E$, whereas in our case $\mathcal{F}_{\infty}^{2}$ contains $\mathcal{F}_{\infty}^{0}, \sigma\left(E_{1}\right)$ and $\sigma\left(E_{2}\right)$. Also, their $\tau_{2}$ does not avoid the $\mathbb{F}^{1}$-stopping times (cf. Proposition 4.3). 


\subsection{Property $(*)$ and the canonical model}

In Sect. 4.1 we saw that the canonical construction yields a loss process $L$ such that the successive $\mathbb{H}$-property $(*)$ holds and $\tau_{1}, \ldots, \tau_{N}$ are $\mathbb{F}^{N}$-totally inaccessible. In this part we prove a converse result: If $\tau_{1}, \ldots, \tau_{N}$ are $\mathbb{F}^{N}$-totally inaccessible and (*) holds, then, under some additional regularity, $L$ must be a canonical loss process. Thus we are back in the general setup of Sect. 2 (i.e., Assumption 4.1 is not made). Note that if a general loss process $L$ admits a predictable compensator of the form $A_{t}=\sum_{n=0}^{N-1} \int_{0}^{t} \mathbf{1}_{\left\{L_{s}=n\right\}} \lambda_{s}^{n} d s$ for some $\mathbb{F}^{n}$-adapted $\lambda^{n} \geq 0$ with $\int_{0}^{t} \mathbf{1}_{\left\{L_{s}=n\right\}} \lambda_{s}^{n} d s<\infty$ a.s. for all $t<\infty$ and $\int_{0}^{\infty} \mathbf{1}_{\left\{L_{s}=n\right\}} \lambda_{s}^{n} d s=\infty$ a.s., $n=0, \ldots, N-1$, then it is a canonical loss process if (and only if) the random variables

$$
E_{n+1}:=\int_{\tau_{n}}^{\tau_{n+1}} \lambda_{s}^{n} d s, \quad n=0, \ldots, N-1
$$

are i.i.d. unit-exponentially distributed and independent of $\mathcal{F}_{\infty}^{0}$ (here, these $E_{1}, \ldots, E_{N}$ are not given a priori). To see this, one can check that by nonnegativity of the $\lambda^{n}$ and since $\tau_{n}<\tau_{n+1}$ a.s., we have indeed

$$
\tau_{n+1}=\inf \left\{t>\tau_{n} \mid \int_{\tau_{n}}^{t} \lambda_{s}^{n} d s \geq \int_{\tau_{n}}^{\tau_{n+1}} \lambda_{s}^{n} d s\right\}=\inf \left\{t>\tau_{n} \mid \int_{\tau_{n}}^{t} \lambda_{s}^{n} d s \geq E_{n+1}\right\}
$$

for all $n=0, \ldots, N-1$. In other words, every canonical loss process can be reconstructed using its $E_{1}, \ldots, E_{N}$ as given in (4.1) in the explicit construction of Definition 4.2 .

The processes $\lambda^{n}$ in (4.1) are only $\mathbb{F}^{n}$-adapted. Thus they "live" beyond $\tau_{n+1}$ because $\tau_{n+1}$ is not $\mathbb{F}^{n}$-adapted, unlike the predictable compensator of $\mathbf{1}_{\left\{\tau_{n+1} \leq t\right\}}$ which remains constant after $\tau_{n+1}$.

The first step is to show existence of the required processes $\lambda^{n}$, which is established in the following Proposition 4.5 without any assumptions beyond absolute continuity and Assumption 2.3.

Proposition 4.5 There exist $\mathbb{F}^{n}$-predictable processes $\Lambda^{n}$ for $n=0, \ldots, N-1$, such that the predictable compensator A of L satisfies

$$
A_{t}=\sum_{n=0}^{N-1} \Lambda_{\tau_{n+1} \wedge t}^{n}-\Lambda_{\tau_{n} \wedge t}^{n}
$$

Moreover, under Assumption 2.3, the $\Lambda^{n}$ above are unique.

If $A$ is absolutely continuous, i.e., $A_{t}=\int_{0}^{t} \lambda_{s} d s$, then for each $n=0, \ldots, N-1$, there exists a unique $\mathbb{F}^{n}$-adapted $\lambda^{n}$ such that

$$
A_{t}=\sum_{n=0}^{N-1} \int_{0}^{t} \mathbf{1}_{\left\{L_{s}=n\right\}} \lambda_{s}^{n} d s
$$

Proof See Appendix B. 
The idea of the proof of Proposition 4.5 is based upon a remark in [8], p. 186, that on the set $\left\{\tau_{n+1} \geq t\right\}$, every $\mathbb{F}^{n+1}$-predictable process is equal to an $\mathbb{F}^{n}$-predictable process, and this process is unique if and only if $\mathbf{P}\left[\tau_{n+1} \leq t \mid \mathcal{F}_{t}^{n}\right]<1$ for all $t \geq 0$.

The standing assumption for the rest of this section is the following:

Assumption 4.6 It holds:

(i) $\mathbb{F}^{0} \stackrel{\mathbb{H}}{\longrightarrow} \mathbb{F}^{N}$.

(ii) $\tau_{n+1}$ avoids the $\mathbb{F}^{n}$-stopping times for all $n=0, \ldots, N-1$, i.e.,

$$
\mathbf{P}\left[\tau_{n+1}=\Theta\right]=0 \quad \text { for every } \mathbb{F}^{n} \text {-stopping time } \Theta .
$$

(iii) $A$ is absolutely continuous and $\lambda^{n}$ in (4.3) satisfies $\int_{0}^{t} \lambda_{s}^{n} d s<\infty$ a.s. for all $t<\infty$ and $\int_{0}^{\infty} \lambda_{s}^{n} d s=\infty$ a.s., for all $n=0, \ldots, N-1$.

Intuitively, (ii) means that when passing from $\mathbb{F}^{n}$ to $\mathbb{F}^{n+1}$, the added random time $\tau_{n+1}$ is "something completely new." Also note that $\int_{0}^{t} \lambda_{s}^{n} d s<\infty$ a.s. in (iii) does not follow from $\mathbf{E}\left[A_{t}\right]=\mathbf{E}\left[L_{t}\right] \leq N<\infty$; it is indeed an additional condition on $\lambda^{n}$.

A useful equivalence to further understand the implications of (ii) is the following:

Lemma 4.7 Let $\mathbb{F}$ be any filtration satisfying the usual conditions and $\tau$ a random time, and let $\mathbb{G}$ be the minimal right-continuous filtration containing $\mathbb{F}$ and making $\tau$ a stopping time. If $\tau$ is $\mathbb{G}$-totally inaccessible, then the following assertions are equivalent:

(i) $\tau$ avoids the $\mathbb{F}$-stopping times, i.e., $\mathbf{P}[\tau=\Theta]=0$ for all $\mathbb{F}$-stopping times $\Theta$.

(ii) The $\mathbb{F}$-martingales do not jump at $\tau$, i.e., $\Delta M_{\tau}=0$ a.s. for all $\mathbb{F}$-martingales $M$.

Proof (i) $\Rightarrow$ (ii): Let (i) hold and $M$ be an $\mathbb{F}$-martingale. We recall that $M$ is càdlàg (has a càdlàg version), hence the stopping times $\Theta_{k}^{\epsilon}$ denoting the times of the $k$ th jump of $M$ larger than $\epsilon$ satisfy $\lim _{k \rightarrow \infty} \Theta_{k}^{\epsilon}=\infty$ a.s. for all $\epsilon>0$. Thus

$$
\mathbf{P}\left[\Delta M_{\tau} \neq 0\right]=\lim _{m \rightarrow \infty} \mathbf{P}\left[\left|\Delta M_{\tau}\right|>1 / m\right]=\lim _{m \rightarrow \infty} \sum_{k=1}^{\infty} \mathbf{P}\left[\tau=\Theta_{k}^{1 / m}\right]=0 .
$$

(ii) $\Rightarrow$ (i): Conversely, assume there exists an $\mathbb{F}$-stopping time $\Theta$ with $\mathbf{P}[\tau=\Theta]>0$. Without loss of generality we may assume that $\Theta$ is $\mathbb{F}$-totally inaccessible. ${ }^{4}$ Then there exists an $\mathbb{F}$-martingale $M$ with exactly one jump, of size one and occurring at $\Theta$ (see [21], Chap. III, Theorem 22, p. 124), and hence

$$
\mathbf{P}\left[\Delta M_{\tau} \neq 0\right]=\mathbf{P}[\tau=\Theta]>0 .
$$

Remark 4.8 It is not possible to directly apply the above Lemma 4.7 to the enlargement from $\mathbb{F}^{0} \vee \mathbb{T}^{1} \vee \cdots \vee \mathbb{T}^{n}$ to $\mathbb{F}^{0} \vee \mathbb{T}^{1} \vee \cdots \vee \mathbb{T}^{n+1}\left(\mathbb{G}^{n}\right.$ to $\mathbb{G}^{n+1}$ ) because, as we

\footnotetext{
${ }^{4}$ By Protter [21] (Chap. III, Theorem 3, p. 104), there exist unique $\mathbb{F}$-stopping times $\Theta_{a}$ and $\Theta_{i}$ such that $\Theta_{a}$ is $\mathbb{F}$-accessible and $\Theta_{i}$ is $\mathbb{F}$-totally inaccessible, $\mathbf{P}\left[\Theta_{a}<\infty, \Theta_{i}<\infty\right]=0$ and $\Theta=\Theta_{a} \wedge \Theta_{i} . \tau<\infty$ a.s. implies $\mathbf{P}[\Theta=\tau]=\mathbf{P}\left[\Theta_{a}=\tau\right]+\mathbf{P}\left[\Theta_{i}=\tau\right]$. Since $\tau$ is $\mathbb{G}$-totally inaccessible, it avoids the $\mathbb{G}$ - and hence the $\mathbb{F}$-accessible stopping times. Therefore $\mathbf{P}\left[\Theta_{a}=\tau\right]=0$ and $\mathbf{P}\left[\Theta_{i}=\tau\right]>0$.
} 
mentioned, $\mathbb{F}^{0} \vee \mathbb{T}^{1} \vee \cdots \vee \mathbb{T}^{n}\left(\mathbb{G}^{n}\right)$ in general does not satisfy the usual conditions (unless $n=0$ or $\mathbb{F}^{0}$ is trivial, see e.g. [21], p. 370). This is one of the reasons why we prefer to work with $\mathbb{F}^{n}$ instead of $\mathbb{F}^{0} \vee \mathbb{T}^{1} \vee \cdots \vee \mathbb{T}^{n}\left(\mathbb{G}^{n}\right)$.

In the rest of this section, we show that every loss process satisfying Assumption 4.6 is a canonical loss process. Given the results above, it remains to show that the $E_{n}$ of (4.1) are indeed i.i.d. unit-exponentially distributed and independent of $\mathcal{F}_{\infty}^{0}$.

Theorem 4.9 Under Assumption 4.6, L is a canonical loss process.

Proof It is well known that under $\mathbb{F}^{n} \stackrel{\mathbb{H}}{\longrightarrow} \mathbb{F}^{n+1}$, the $\mathbb{F}^{n}$-supermartingale

$$
G_{t}^{n}:=\mathbf{P}\left[\tau_{n+1}>t \mid \mathcal{F}_{t}^{n}\right] \stackrel{\mathbb{H}}{=} \mathbf{P}\left[\tau_{n+1}>t \mid \mathcal{F}_{\infty}^{n}\right]
$$

is nonincreasing and $\mathbb{F}^{n}$-predictable. Due to "the key lemma" 3.5 in [2] (see also Lemma 5.2 below for a generalization), the relationship between $G_{t}^{n}$ and $\Lambda_{t}^{n}$, respectively $\lambda_{t}^{n}$, is that

$$
\frac{d G_{t}^{n}}{G_{t-}^{n}}=-d \Lambda_{t \wedge \tau_{n}}^{n}=-\mathbf{1}_{\left\{\tau_{n} \leq t\right\}} \lambda_{t}^{n} d t .
$$

Then $\int_{0}^{t} \lambda_{s}^{n} d s<\infty$ a.s. for all $t<\infty$ implies $\Gamma_{t}^{n}:=-\log G_{t}^{n}=\Lambda_{t}^{n}-\Lambda_{t \wedge \tau_{n}}^{n}=$ $\int_{t \wedge \tau_{n}}^{t} \lambda_{s}^{n} d s$. See e.g. [3] and the references therein for these results.

It has to be noted next that under $\mathbb{F}^{n} \stackrel{\mathbb{H}}{\longrightarrow} \mathbb{F}^{n+1}, G^{n}$ is continuous if and only if $\tau_{n+1}$ avoids the $\mathbb{F}^{n}$-stopping times. Hence, according to the proof of Lemma 2 in [3], since $G^{n}$ is continuous and $\int_{0}^{\infty} \lambda_{s}^{n} d s=\infty$ a.s., $E_{n+1}:=\Gamma_{\tau_{n+1}}^{n}$ is indeed unitexponentially distributed and independent of $\mathcal{F}_{\infty}^{n}$ for each $n=1, \ldots, N$.

Further, since $E_{n}$ is $\mathcal{F}_{\infty}^{n}$-measurable for every $n=1, \ldots, N$, the variables $E_{1}, \ldots, E_{N}$ are also independent. A direct proof of this result can be found in Theorem 44 of [9].

\section{The conditional Markov loss model}

Now that we have a concrete stochastic representation of the loss process, we can tackle some of the pricing problems that were motivated in Sect. 2.1. There, one important step was in (2.1) which tried to express $\mathbf{1}_{\left\{L_{t}=k\right\}} P_{n}(t, T)=\mathbf{1}_{\left\{L_{t}=k\right\}} \widetilde{P}_{n, k}(t, T)$ for some suitably defined, $\mathbb{F}^{0}$-adapted $\widetilde{P}_{n, k}(t, T)$. The ultimate goal was to be able to move from expressions conditional on the market filtration $\mathbb{F}^{N}$ to expressions conditional only on the background filtration $\mathbb{F}^{0}$.

5.1 A general Bayes-type rule

Definition 5.1 The $\mathcal{F}_{t}^{n}$-conditional transition probabilities are defined as

$$
\widehat{P}_{n, m}(t, T):=\mathbf{1}_{\left\{L_{t} \geq n\right\}} \frac{\mathbf{E}\left[\mathbf{1}_{\left\{L_{t}=n\right\}} \mathbf{1}_{\left\{L_{T}=m\right\}} \mid \mathcal{F}_{t}^{n}\right]}{\mathbf{E}\left[\mathbf{1}_{\left\{L_{t}=n\right\}} \mid \mathcal{F}_{t}^{n}\right]} .
$$


By Bayes' rule, $\widehat{P}_{n, m}(t, T)$ can be regarded as the probability of going to $L_{T}=m$, conditionally on $L_{t}=n$ and $\mathcal{F}_{t}^{n}$. Note that $\left\{L_{t}=n\right\}=\left\{L_{t} \geq n\right\} \cap\left\{L_{t} \leq n\right\}$ is not $\mathcal{F}_{t}^{n}$ measurable. Indeed, on $\mathbb{F}^{n}$, we observe $\tau_{n}$ but we do not know if $L$ has moved on in the meantime; more precisely $\mathbf{1}_{\left\{L_{t} \geq n\right\}}=\mathbf{1}_{\left\{\tau_{n} \leq t\right\}}$ is $\mathbb{F}^{n}$-adapted, but $\mathbf{1}_{\left\{L_{t} \leq n\right\}}=\mathbf{1}_{\left\{\tau_{n+1}>t\right\}}$ is not. Thus

$$
\mathbf{E}\left[\mathbf{1}_{\left\{L_{t}=n\right\}} \mid \mathcal{F}_{t}^{n}\right]=\mathbf{1}_{\left\{L_{t} \geq n\right\}} \mathbf{E}\left[\mathbf{1}_{\left\{\tau_{n+1}>t\right\}} \mid \mathcal{F}_{t}^{n}\right],
$$

and by Assumption 2.3 the conditional expectation on the right-hand side is (strictly) positive on the set $\left\{L_{t} \geq n\right\}$.

The following lemma holds irrespective of any $\mathbb{H}$-assumption on the consecutive enlargement $\mathbb{F}^{0} \subseteq \cdots \subseteq \mathbb{F}^{N}$ and tells how close we can get to (2.1).

Lemma 5.2 For any $X \in L^{1}\left(\mathcal{F}_{T}^{n}\right)$ we have

$$
\mathbf{1}_{\left\{L_{t}=n\right\}} \mathbf{E}\left[X \mathbf{1}_{\left\{L_{T}=m\right\}} \mid \mathcal{F}_{t}^{N}\right]=\mathbf{1}_{\left\{L_{t}=n\right\}} \frac{\mathbf{E}\left[X \mathbf{1}_{\left\{L_{t}=n\right\}} \mathbf{1}_{\left\{L_{T}=m\right\}} \mid \mathcal{F}_{t}^{n}\right]}{\mathbf{E}\left[\mathbf{1}_{\left\{L_{t}=n\right\}} \mid \mathcal{F}_{t}^{n}\right]} .
$$

In particular,

$$
\mathbf{1}_{\left\{L_{t}=n\right\}} P_{m}(t, T)=\mathbf{1}_{\left\{L_{t}=n\right\}} \widehat{P}_{n, m}(t, T) .
$$

Proof See Appendix C.

For the pure conditional survival probability, the difference of (5.2) to our aim of (2.1) is that $\widehat{P}_{n, m}(t, T)$ is $\mathcal{F}_{t}^{n}$-measurable, while we desired $\widetilde{P}_{n, m}(t, T)$ which are $\mathcal{F}_{t}^{0}$-measurable, i.e., purely background-measurable.

Remark 5.3 For $m=n=1,(5.2)$ is already well known; it can be found e.g. in [3], Lemma 1 (p. 147).

5.2 The conditional Markov model, a special case

Note that if $L$ is a canonical loss process, then for $m=n$, since $\mathbf{1}_{\left\{L_{t}=n\right\}} \mathbf{1}_{\left\{L_{T}=n\right\}}=$ $\mathbf{1}_{\left\{L_{t} \geq n\right\}} \mathbf{1}_{\left\{\tau_{n+1}>T\right\}}$ for all $T \geq t$, we have by Proposition 4.3, P2 that

$$
\mathbf{1}_{\left\{L_{t}=n\right\}} \widehat{P}_{n, n}(t, T)=\mathbf{1}_{\left\{L_{t}=n\right\}} \frac{\mathbf{E}\left[\mathbf{E}\left[\mathbf{1}_{\left\{\tau_{n+1}>T\right\}} \mid \mathcal{F}_{T}^{n}\right] \mid \mathcal{F}_{t}^{n}\right]}{\mathbf{E}\left[\mathbf{1}_{\left\{\tau_{n+1}>t\right\}} \mid \mathcal{F}_{t}^{n}\right]}=\mathbf{1}_{\left\{L_{t}=n\right\}} \mathbf{E}\left[e^{-\int_{t}^{T} \lambda_{s}^{n} d s} \mid \mathcal{F}_{t}^{n}\right] .
$$

Using in addition that a canonical loss process has the successive $\mathbb{H}$-property, we observe that if $\lambda^{n}$ were $\mathbb{F}^{0}$-adapted, then on the set $\left\{L_{t}=n\right\}, \widehat{P}_{n, n}(t, T)$-and so $P_{n}(t, T)$-would indeed be equal to the $\mathcal{F}_{t}^{0}$-measurable variable

$$
\mathbf{E}\left[e^{-\int_{t}^{T} \lambda_{s}^{n} d s} \mid \mathcal{F}_{t}^{n}\right] \stackrel{\mathbb{H}}{=} \mathbf{E}\left[e^{-\int_{t}^{T} \lambda_{s}^{n} d s} \mid \mathcal{F}_{t}^{0}\right]
$$

which is what we are after. Therefore, in order to find a decomposition (2.1) we make the following assumption. 
Assumption 5.4 The loss process is a canonical loss process, and the processes $\lambda^{n}$ in (4.1) are $\mathbb{F}^{0}$-adapted for all $n=0, \ldots, N-1$.

In this case, $L$ can be interpreted as a finite-state Markov chain if it is conditioned on $\mathcal{F}_{\infty}^{0}$. This assumption does not rule out contagion, as the intensity of $L$ still can depend on the level of $L$ itself. Assumption 5.4 should not be confused with the alternative assumption that the loss process and its intensity $(L, \lambda)$ jointly form a Markov process. The latter assumption is made e.g. in [14] and [13].

Definition 5.5 The $\mathcal{F}_{\infty}^{0}$ - and $\mathcal{F}_{t}^{0}$-conditional transition probabilities are defined by

$$
\begin{aligned}
\widetilde{P}_{n, m}(t, T) & :=\frac{\mathbf{E}\left[\mathbf{1}_{\left\{L_{t}=n\right\}} \mathbf{1}_{\left\{L_{T}=m\right\}} \mid \mathcal{F}_{t}^{0}\right]}{\mathbf{E}\left[\mathbf{1}_{\left\{L_{t}=n\right\}} \mid \mathcal{F}_{t}^{0}\right]}, \\
\widetilde{P}_{n, m}^{\infty}(t, T) & :=\frac{\mathbf{E}\left[\mathbf{1}_{\left\{L_{t}=n\right\}} \mathbf{1}_{\left\{L_{T}=m\right\}} \mid \mathcal{F}_{\infty}^{0}\right]}{\mathbf{E}\left[\mathbf{1}_{\left\{L_{t}=n\right\}} \mid \mathcal{F}_{\infty}^{0}\right]}, \\
\widetilde{P}_{n}(t) & :=\mathbf{E}\left[\mathbf{1}_{\left\{L_{t}=n\right\}} \mid \mathcal{F}_{t}^{0}\right] \stackrel{\mathbb{H}}{=} \mathbf{E}\left[\mathbf{1}_{\left\{L_{t}=n\right\}} \mid \mathcal{F}_{\infty}^{0}\right] .
\end{aligned}
$$

We begin with an intuitive result, which is due to the interpretation of $L$ as an $\mathcal{F}_{\infty}^{0}$-conditional Markov chain.

Lemma 5.6 The $\mathcal{F}_{\infty}^{0}$-conditional transition probabilities can be represented as

$$
\widetilde{P}_{n, m}^{\infty}(t, T)=\mathbf{1}_{\{m=n\}} e^{-\int_{t}^{T} \lambda_{s}^{n} d s}+\int_{t}^{T} e^{-\int_{u}^{T} \lambda_{s}^{m} d s} \widetilde{P}_{n, m-1}^{\infty}(t, u) \lambda_{u}^{m-1} d u,
$$

and

$$
\mathbf{1}_{\left\{L_{t} \geq n\right\}} \widetilde{P}_{n, m}^{\infty}(t, T)=\mathbf{1}_{\left\{L_{t} \geq t\right\}} \frac{\mathbf{E}\left[\mathbf{1}_{\left\{L_{t}=n\right\}} \mathbf{1}_{\left\{L_{T}=m\right\}} \mid \mathcal{F}_{\infty}^{n}\right]}{\mathbf{E}\left[\mathbf{1}_{\left\{L_{t}=n\right\}} \mid \mathcal{F}_{\infty}^{n}\right]}
$$

Proof See Appendix D.

The formulae for $\widehat{P}_{n, m}(t, T)$ now follow directly:

\section{Corollary 5.7 It holds}

$$
\begin{aligned}
\widetilde{P}_{n, m}(t, T)= & \mathbf{E}\left[\widetilde{P}_{n, m}^{\infty}(t, T) \mid \mathcal{F}_{t}^{0}\right] \\
= & \mathbf{1}_{\{m=n\}} \mathbf{E}\left[e^{-\int_{t}^{T} \lambda_{s}^{n} d s} \mid \mathcal{F}_{t}^{0}\right] \\
& +\int_{t}^{T} \mathbf{E}\left[e^{-\int_{u}^{T} \lambda_{s}^{m} d s} \widetilde{P}_{n, m-1}^{\infty}(t, u) \lambda_{u}^{m-1} \mid \mathcal{F}_{t}^{0}\right] d u,
\end{aligned}
$$

and we have the relationship

$$
\widehat{P}_{n, m}(t, T)=\mathbf{1}_{\left\{L_{t} \geq n\right\}} \widetilde{P}_{n, m}(t, T) .
$$


Lemma 5.6 and its corollary also show that $\widetilde{P}_{n, m}^{\infty}(t, T)$ is continuous and $\widetilde{P}_{n, m}(t, T)$ is right-continuous in $T$ a.s.

Proof of Corollary 5.7 The formula for $\widetilde{P}_{n, m}(t, T)$ follows immediately from Lemma 5.6 and

$$
\begin{aligned}
\widetilde{P}_{n, m}(t, T) & =\mathbf{E}\left[\frac{\mathbf{E}\left[\mathbf{1}_{\left\{L_{t}=n\right\}} \mathbf{1}_{\left\{L_{T}=m\right\}} \mid \mathcal{F}_{\infty}^{0}\right]}{\mathbf{E}\left[\mathbf{1}_{\left\{L_{t}=n\right\}} \mid \mathcal{F}_{t}^{0}\right]} \mid \mathcal{F}_{t}^{0}\right] \\
& \stackrel{\mathbb{H}}{=} \mathbf{E}\left[\frac{\mathbf{E}\left[\mathbf{1}_{\left\{L_{t}=n\right\}} \mathbf{1}_{\left\{L_{T}=m\right\}} \mid \mathcal{F}_{\infty}^{0}\right]}{\mathbf{E}\left[\mathbf{1}_{\left\{L_{t}=n\right\}} \mid \mathcal{F}_{\infty}^{0}\right]} \mid \mathcal{F}_{t}^{0}\right]=\mathbf{E}\left[\widetilde{P}_{n, m}^{\infty}(t, T) \mid \mathcal{F}_{t}^{0}\right],
\end{aligned}
$$

and then the relationship $\widehat{P}_{n, m}(t, T)=\mathbf{1}_{\left\{L_{t} \geq n\right\}} \widetilde{P}_{n, m}(t, T)$ is due to

$$
\begin{aligned}
\widehat{P}_{n, m}(t, T) & \stackrel{\mathbb{H}}{=} \mathbf{E}\left[\mathbf{1}_{\left\{L_{t} \geq t\right\}} \frac{\mathbf{E}\left[\mathbf{1}_{\left\{L_{t}=n\right\}} \mathbf{1}_{\left\{L_{T}=m\right\}} \mid \mathcal{F}_{\infty}^{n}\right]}{\mathbf{E}\left[\mathbf{1}_{\left\{L_{t}=n\right\}} \mid \mathcal{F}_{\infty}^{n}\right]} \mid \mathcal{F}_{t}^{n}\right] \\
& =\mathbf{1}_{\left\{L_{t} \geq n\right\}} \mathbf{E}\left[\widetilde{P}_{n, m}^{\infty}(t, T) \mid \mathcal{F}_{t}^{n}\right] \\
& \stackrel{\mathbb{H}}{=} \mathbf{1}_{\left\{L_{t} \geq n\right\}} \mathbf{E}\left[\widetilde{P}_{n, m}^{\infty}(t, T) \mid \mathcal{F}_{t}^{0}\right]=\mathbf{1}_{\left\{L_{t} \geq n\right\}} \widetilde{P}_{n, m}(t, T) .
\end{aligned}
$$

A consequence of Lemma 5.6 and its corollary is that in the conditional Markov setup we can represent the market-filtration loss probabilities $P_{m}$ as initially indicated in (2.1), or

$$
P_{m}(t, T)=\sum_{n=0}^{N} \mathbf{1}_{\left\{L_{t}=n\right\}} \widetilde{P}_{n, m}(t, T) .
$$

This means that restricted to any set $\left\{L_{t}=n\right\}$, the loss probabilities $P_{m}$ depend only on the "default-free" information $\mathcal{F}_{t}^{0}$.

Remark 5.8 As an aside of Lemma 5.6 we also observe the $\mathcal{F}_{\infty}^{0}$-conditional Kolmogorov forward ODE

$$
\partial_{T} \underline{\widetilde{P}}^{\infty}(t, T)=\underline{\widetilde{P}}^{\infty}(t, T) \underline{\lambda}(T), \quad \underline{\widetilde{P}}^{\infty}(t, t)=\mathrm{id}_{N+1},
$$

where $\underline{\widetilde{P}}^{\infty}(t, T)$ is the matrix with $\underline{\widetilde{P}}^{\infty}(t, T)_{n, m}:=\widetilde{P}_{n, m}^{\infty}(t, T), \underline{\lambda}(T)$ is the generator matrix with $\underline{\lambda}(T)_{n, m}=-\mathbf{1}_{\{n=m\}} \lambda_{T}^{n}+\mathbf{1}_{\{m=n+1\}} \lambda_{T}^{n}$, and $\operatorname{id}_{k}$ is an identity matrix of dimension $k$.

Finally, we give a formal proof that $L$ is indeed a Markov chain if conditioned on $\mathcal{F}_{\infty}^{0}$.

Lemma 5.9 For every $k>0$ and every $0 \leq u_{1} \leq \cdots \leq u_{k}<t$ and $n_{1} \leq \cdots \leq n_{k} \leq n$, it holds that

$$
\frac{\mathbf{E}\left[\mathbf{1}_{\left\{L_{T}=m\right\}} \mathbf{1}_{\left\{L_{t}=n\right\}} \mathbf{1}_{\left\{L_{u_{k}}=n_{k}\right\}} \cdots \mathbf{1}_{\left\{L_{u_{1}}=n_{1}\right\}} \mid \mathcal{F}_{\infty}^{0}\right]}{\mathbf{E}\left[\mathbf{1}_{\left\{L_{t}=n\right\}} \mathbf{1}_{\left\{L_{u_{k}}=n_{k}\right\}} \cdots \mathbf{1}_{\left\{L_{u_{1}}=n_{1}\right\}} \mid \mathcal{F}_{\infty}^{0}\right]}=\widetilde{P}_{n, m}^{\infty}(t, T) .
$$


Proof We distinguish the cases $n_{k}<n$ and $n_{k}=n$. For $n_{k}<n$, the numerator on the left-hand side simplifies to

$$
\begin{aligned}
\mathbf{E} & {\left[\mathbf{1}_{\left\{L_{T}=m\right\}} \mathbf{1}_{\left\{L_{t}=n\right\}} \mathbf{1}_{\left\{L_{u_{k}}=n_{k}\right\}} \cdots \mathbf{1}_{\left\{L_{u_{1}}=n_{1}\right\}} \mid \mathcal{F}_{\infty}^{0}\right] } \\
& =\mathbf{E}\left[\mathbf{E}\left[\mathbf{1}_{\left\{L_{T}=m\right\}} \mathbf{1}_{\left\{L_{t}=n\right\}} \mid \mathcal{F}_{\infty}^{n}\right] \mathbf{1}_{\left\{L_{u_{k}}=n_{k}\right\}} \cdots \mathbf{1}_{\left\{L_{u_{1}}=n_{1}\right\}} \mid \mathcal{F}_{\infty}^{0}\right] \\
& =\widetilde{P}_{n, m}^{\infty}(t, T) \mathbf{E}\left[\mathbf{E}\left[\mathbf{1}_{\left\{L_{t}=n\right\}} \mid \mathcal{F}_{\infty}^{n}\right] \mathbf{1}_{\left\{L_{u_{k}}=n_{k}\right\}} \cdots \mathbf{1}_{\left\{L_{u_{1}}=n_{1}\right\}} \mid \mathcal{F}_{\infty}^{0}\right],
\end{aligned}
$$

and the denominator satisfies

$$
\begin{aligned}
& \mathbf{E}\left[\mathbf{1}_{\left\{L_{t}=n\right\}} \mathbf{1}_{\left\{L_{u_{k}}=n_{k}\right\}} \cdots \mathbf{1}_{\left\{L_{u_{1}}=n_{1}\right\}} \mid \mathcal{F}_{\infty}^{0}\right] \\
& \quad=\mathbf{E}\left[\mathbf{E}\left[\mathbf{1}_{\left\{L_{t}=n\right\}} \mid \mathcal{F}_{\infty}^{n}\right]_{\left\{L_{u_{k}}=n_{k}\right\}} \cdots \mathbf{1}_{\left\{L_{u_{1}}=n_{1}\right\}} \mid \mathcal{F}_{\infty}^{0}\right] .
\end{aligned}
$$

In the case $n_{k-1}<n_{k}=n$, we notice that on the set $\left\{L_{t}=n\right\}$, we have $\mathbf{1}_{\left\{L_{u_{k}}=n_{k}\right\}}=\mathbf{1}_{\left\{L_{u_{k}} \geq n\right\}}$ which is $\mathcal{F}_{t}^{n}$-measurable. Then the proof is analogous. We do not need to consider the case $n_{k-1}=n_{k}=n$ since $\mathbf{1}_{\left\{L_{u_{k-1}}=n\right\}} \mathbf{1}_{\left\{L_{u_{k}}=n\right\}} \mathbf{1}_{\left\{L_{t}=n\right\}}=$ $\mathbf{1}_{\left\{L_{u_{k-1}}=n\right\}} \mathbf{1}_{\left\{L_{t}=n\right\}}$.

\subsection{Conclusion}

We can now give answers to the questions that were raised at the end of Sect. 2.1.

The convenient representation of the loss probabilities given in (2.1), which allowed the payoff decomposition and the transformation of the pricing problem, holds under Assumption 5.4, i.e., if the loss process satisfies the successive $\mathbb{H}$-property and if the intensity processes in its canonical representation are all $\mathbb{F}^{0}$-adapted.

Then, Corollary 5.7 gives the expressions that must be evaluated in order to derive $\widetilde{P}_{n, m}(t, T)$ and of course also $\widetilde{P}_{n, m}(0, t)$. For this, the Kolmogorov-type ordinary differential equations given in Remark 5.8 may also be helpful, but concrete values for $\widetilde{P}_{n, m}(t, T)$ will of course always depend on the specification of the dynamics of the $\lambda_{t}^{n}$. Nevertheless, none of the resulting pricing expressions contains a direct reference to the loss process $L$ itself.

\section{Appendix A: Proof of Proposition 4.3}

Proof of Proposition 4.3 P1: Since $\tau_{0}=0$ a.s., we may assume we have shown $\tau_{n}<\infty$ a.s. Then

$$
\begin{aligned}
\mathbf{P}\left[\tau_{n}<\tau_{n+1}<\infty\right] & =\lim _{m \rightarrow \infty} \mathbf{P}\left[\tau_{n}+1 / m<\tau_{n+1} \leq m\right] \\
& =\lim _{m \rightarrow \infty} \mathbf{P}\left[\int_{\tau_{n}}^{\tau_{n}+1 / m} \lambda_{s}^{n} d s<E_{n+1} \leq \int_{\tau_{n} \wedge m}^{1 / m} \lambda_{s}^{n} d s\right] \\
& =\mathbf{P}\left[0<E_{n+1}<\infty\right]=1
\end{aligned}
$$

which implies P1.

We continue with an auxiliary result. Its proof can be found below. 
Lemma A.1 $E_{n+1}, \ldots, E_{N}$ are independent of $\mathcal{F}_{\infty}^{n}$ for each $n=0, \ldots, N-1$.

Proof of Proposition 4.3 (continued). P2: Using Lemma A.1, we find first

$$
\mathbf{P}\left[\tau_{n+1}>t \mid \mathcal{F}_{\infty}^{n}\right]=\mathbf{P}\left[E_{n+1}>\int_{t \wedge \tau_{n}}^{t} \lambda_{s}^{n} d s \mid \mathcal{F}_{\infty}^{n}\right]=e^{-\int_{t \wedge \tau_{n}}^{t} \lambda_{s}^{n} d s}
$$

P3: Second, since $\mathbf{P}\left[\tau_{n+1}>t \mid \mathcal{F}_{\infty}^{n}\right]$ is $\mathcal{F}_{t}^{n}$-measurable, it is equal to $\mathbf{P}\left[\tau_{n+1}>t \mid \mathcal{F}_{t}^{n}\right]$ and it is well known that in our setup this is equivalent to $\mathbb{F}^{n} \stackrel{\mathbb{H}}{\longrightarrow} \mathbb{F}^{n+1}$ (see e.g. [7], p. 71, or [1], Sect. 6.1.1). ${ }^{5}$

P4: By property P3 it is sufficient to show that $A_{t}^{n+1}:=\int_{0}^{t} \mathbf{1}_{\left\{L_{s}=n\right\}} \lambda_{s}^{n} d s$ is the predictable $\mathbb{F}^{n+1}$-compensator of $\mathbf{1}_{\left\{\tau_{n+1} \leq t\right\}}$. Using localization, we may assume without restriction that $A_{t}^{n+1}$ is bounded. Further, due to Lemma 3.6 (i) and since $\mathbf{1}_{\left\{\tau_{n+1} \leq t\right\}}-A_{t}^{n+1}$ is càdlàg (and locally bounded), it is sufficient to show that $A^{n+1}$ is the predictable ( $\mathbb{F}^{n} \vee \mathbb{T}^{n+1}$ )-compensator of $\mathbf{1}_{\left\{\tau_{n+1} \leq t\right\}}$. Obviously, $A^{n+1}$ is nondecreasing and $\mathbb{F}^{n+1}$-predictable (continuous and $\mathbb{F}^{n+1}$-adapted). It remains to show the martingale property. Take $s<t$ and $F:=F^{n} \cap\left\{\tau_{n+1}>u\right\}$ for some $F^{n} \in \mathcal{F}_{s}^{n}$ and $u \leq s$. Then $\mathbf{1}_{F} \mathbf{1}_{\left\{\tau_{n+1}>s\right\}}=\mathbf{1}_{F^{n}} \mathbf{1}_{\left\{\tau_{n+1}>s\right\}}$. Using repeatedly P2, we deduce

$$
\begin{aligned}
\mathbf{E}\left[\mathbf{1}_{F}\left(\mathbf{1}_{\left\{\tau_{n+1} \leq t\right\}}-\mathbf{1}_{\left\{\tau_{n+1} \leq s\right\}}\right)\right] & =\mathbf{E}\left[\mathbf{1}_{F^{n}}\left(\mathbf{1}_{\left\{\tau_{n+1}>s\right\}}-\mathbf{1}_{\left\{\tau_{n+1}>t\right\}}\right)\right] \\
& =\mathbf{E}\left[\mathbf{1}_{F^{n}}\left(e^{-\int_{s \wedge \tau_{n}}^{s} \lambda_{v}^{n} d v}-e^{-\int_{t \wedge \tau_{n}}^{t} \lambda_{v}^{n} d v}\right)\right] \\
& =\mathbf{E}\left[\mathbf{1}_{F^{n}} \int_{s}^{t} e^{-\int_{y \wedge \tau_{n}}^{y} \lambda_{v}^{n} d v} \mathbf{1}_{\left\{\tau_{n} \leq y\right\}} \lambda_{y}^{n} d y\right] \\
& =\int_{s}^{t} \mathbf{E}\left[\mathbf{1}_{F^{n}} \mathbf{1}_{\left\{\tau_{n+1}>y\right\}} \mathbf{1}_{\left\{\tau_{n} \leq y\right\}} \lambda_{y}^{n}\right] d y \\
& =\mathbf{E}\left[\mathbf{1}_{F^{n}} \mathbf{1}_{\left\{\tau_{n+1}>s\right\}} \int_{s}^{t} \mathbf{1}_{\left\{L_{y}=n\right\}} \lambda_{y}^{n} d y\right] \\
& =\mathbf{E}\left[\mathbf{1}_{F}\left(A_{t}^{n+1}-A_{s}^{n+1}\right)\right] .
\end{aligned}
$$

By Dynkin's lemma, this holds for every $F \in \mathcal{F}_{s}^{n} \vee \mathcal{T}_{s}^{n+1}$ because $\mathcal{F}_{s}^{n} \vee \mathcal{T}_{s}^{n+1}$ is generated by sets of the form $F^{n} \cap\left\{\tau_{n+1}>u\right\}$ with $F^{n} \in \mathcal{F}_{s}^{n}$ and $u \leq s$.

P5: Let $\Theta$ be an $\mathbb{F}^{n}$-stopping time; then $\Theta=\tau_{n+1}$ implies $\int_{\tau_{n}}^{\Theta} \lambda_{s}^{n} d s=E_{n+1}$. Hence

$$
\mathbf{P}\left[\tau_{n+1}=\Theta\right] \leq \mathbf{P}\left[\int_{\Theta \wedge \tau_{n}}^{\Theta} \lambda_{s}^{n} d s=E_{n+1}\right]=\mathbf{E}\left[\mathbf{P}\left[\int_{\Theta \wedge \tau_{n}}^{\Theta} \lambda_{s}^{n} d s=E_{n+1} \mid \mathcal{F}_{\infty}^{n}\right]\right]=0
$$

because $E_{n+1}$ has a diffuse law and is independent of $\mathcal{F}_{\infty}^{n}$ (again by Lemma A.1).

\footnotetext{
${ }^{5}$ Bielecki and Rutkowski [1] only show equivalence to $\mathbb{F}^{n} \stackrel{\mathbb{H}}{\longrightarrow} \mathbb{F}^{n} \vee \mathbb{T}^{n+1}$. One can then pass from $\mathbb{F}^{n} \vee \mathbb{T}^{n+1}$ to $\mathbb{F}^{n+1}$ by the right-continuity of $\mathbb{F}^{n}$ as in Lemma 3.6 (ii).
} 
Proof of Lemma A.1 For $n=0$ the claim is trivially satisfied. We (may) continue assuming the claim holds for $n<N-1$, i.e.,

$$
\mathbf{P}\left[\bigcap_{k=n+1}^{N}\left\{E_{k}>x_{k}\right\} \cap F\right]=\mathbf{P}[F] \prod_{k=n+1}^{N} e^{-x_{k}}
$$

for all $x_{n+1}, \ldots, x_{N} \geq 0$ and $F \in \mathcal{F}_{\infty}^{n}$. We want to show that the claim holds for $n+1$. To that end, let $F:=\left\{\underline{\tau}^{n+1}>\underline{u}^{n+1}\right\} \cap F^{0}$ with $\underline{u} \in[0, \infty)^{N}$ and $F^{0} \in \mathcal{F}_{\infty}^{0}$ and define $S_{y}:=\int_{y \wedge \tau_{n}}^{y} \lambda_{s}^{n} d s, y \geq 0$. Then $\left\{\tau_{n+1}>u_{n+1}\right\}=\left\{E_{n+1}>S_{u_{n+1}}\right\}$ and, since $S_{u_{n+1}}$ is nonnegative and $\mathcal{F}_{\infty}^{n}$-measurable, there exist $x_{1}^{m}, \ldots, x_{m}^{m} \geq 0$ and disjoint $F_{1}^{m}, \ldots, F_{m}^{m} \in \mathcal{F}_{\infty}^{n}$, for each $m \in \mathbb{N}$, such that $\sum_{j=1}^{m} x_{j}^{m} \mathbf{1}_{F_{j}^{m}} \uparrow S_{u_{n+1}}{ }^{6}$ Note that $F^{n}:=\left\{\underline{\tau}^{n}>\underline{u}^{n}\right\} \cap F^{0} \in \mathcal{F}_{\infty}^{n}$. Thus

$$
\begin{aligned}
& \mathbf{P}\left[\bigcap_{k=n+2}^{N}\left\{E_{k}>x_{k}\right\} \cap F\right] \\
& =\mathbf{P}\left[\bigcap_{k=n+2}^{N}\left\{E_{k}>x_{k}\right\} \cap\left\{E_{n+1}>S_{u_{n+1}}\right\} \cap F^{n}\right] \\
& =\lim _{m \rightarrow \infty} \sum_{j=1}^{m} \mathbf{P}\left[\bigcap_{k=n+2}^{N}\left\{E_{k}>x_{k}\right\} \cap\left\{E_{n+1}>x_{j}^{m}\right\} \cap F_{j}^{m} \cap F^{n}\right] \\
& =\lim _{m \rightarrow \infty} \sum_{j=1}^{m} \mathbf{P}\left[F_{j}^{m} \cap F^{n}\right] e^{-x_{j}^{m}} \prod_{k=n+2}^{N} e^{-x_{k}} \\
& =\lim _{m \rightarrow \infty} \sum_{j=1}^{m} \mathbf{P}\left[\left\{E_{n+1}>x_{j}^{m}\right\} \cap F_{j}^{m} \cap F^{n}\right] \prod_{k=n+2}^{N} e^{-x_{k}} \\
& =\mathbf{P}\left[\left\{E_{n+1}>S_{u_{n+1}}\right\} \cap F^{n}\right] \prod_{k=n+2}^{N} e^{-x_{k}}=\mathbf{P}[F] \prod_{k=n+2}^{N} e^{-x_{k}},
\end{aligned}
$$

i.e., the claim holds for $n+1$ because $\mathcal{F}_{\infty}^{n+1}$ is generated by sets of the form like $F$.

\section{Appendix B: Proof of Proposition 4.5}

Proof of Proposition 4.5 By [8], p. 186, there exists an $\mathbb{F}^{N-1}$-predictable process $\Lambda^{N-1}$ with

$$
\mathbf{1}_{\left\{t \leq \tau_{N}\right\}}\left(\Lambda_{t}^{N-1}-\Lambda_{t}\right)=0 .
$$

${ }^{6}$ See e.g. the proof of Theorem 4.13 in [25]. 
In particular, this shows also that $\Lambda_{\tau_{N}}^{N-1}=\Lambda_{\tau_{N}}$ on $\left\{\tau_{N}<\infty\right\}$. Clearly, $\Lambda_{t}=\Lambda_{\tau_{N} \wedge t}$ (because $L_{t}=L_{\tau_{N} \wedge t}$ ) and thus

$$
\Lambda_{t}=\mathbf{1}_{\left\{t \leq \tau_{N}\right\}} \Lambda_{t}+\mathbf{1}_{\left\{t>\tau_{N}\right\}} \Lambda_{\tau_{N}}=\mathbf{1}_{\left\{t \leq \tau_{N}\right\}} \Lambda_{t}^{N-1}+\mathbf{1}_{\left\{t>\tau_{N}\right\}} \Lambda_{\tau_{N}}^{N-1}=\Lambda_{\tau_{N} \wedge t}^{N-1},
$$

which proves the claim for $N=1$. For $N>1$ we may continue by induction, assuming we have shown that there exist $\mathbb{F}^{n}$-predictable $\Lambda^{n}$ for $n=k, \ldots, N-1$ such that

$$
\Lambda_{t}=\Lambda_{\tau_{k+1} \wedge t}^{k}+\sum_{n=k+1}^{N-1} \Lambda_{\tau_{n+1} \wedge t}^{n}-\Lambda_{\tau_{n} \wedge t}^{n} .
$$

Then the $\mathbb{F}^{N}$-compensator of $L_{\tau_{k} \wedge t}$ is $\Lambda_{\tau_{k} \wedge t}=\Lambda_{\tau_{k} \wedge t}^{k}$. Again, by the argument stated above, there exists an $\mathbb{F}^{k-1}$-predictable process $\Lambda^{k-1}$ with $\mathbf{1}_{\left\{t \leq \tau_{k}\right\}}\left(\Lambda_{t}^{k-1}-\Lambda_{t}^{k}\right)=0$ and hence $\Lambda_{\tau_{k}}^{k-1}=\Lambda_{\tau_{k}}^{k}$ on $\left\{\tau_{k}<\infty\right\}$, which implies

$$
\begin{aligned}
\Lambda_{t} & =\Lambda_{\tau_{k+1} \wedge t}^{k}+\sum_{n=k+1}^{N-1} \Lambda_{\tau_{n+1} \wedge t}^{n}-\Lambda_{\tau_{n} \wedge t}^{n} \\
& =\mathbf{1}_{\left\{t \leq \tau_{k}\right\}} \Lambda_{\tau_{k} \wedge t}^{k}+\mathbf{1}_{\left\{t>\tau_{k}\right\}} \Lambda_{\tau_{k}}^{k}+\left(\Lambda_{\tau_{k+1} \wedge t}^{k}-\Lambda_{\tau_{k} \wedge t}^{k}\right)+\sum_{n=k+1}^{N-1} \Lambda_{\tau_{n+1} \wedge t}^{n}-\Lambda_{\tau_{n} \wedge t}^{n} \\
& =\Lambda_{\tau_{k} \wedge t}^{k-1}+\sum_{n=k}^{N-1} \Lambda_{\tau_{n+1} \wedge t}^{n}-\Lambda_{\tau_{n} \wedge t}^{n} .
\end{aligned}
$$

Repeating this step until $k=1$ proves existence. Further, as shown in [8], p. 186, each $\Lambda^{k-1}$ is unique if (and only if) $\mathbf{P}\left[\tau_{k} \leq t \mid \mathcal{F}_{t}^{k-1}\right]<1$ for all $t \geq 0$. Equation (4.3) now follows directly.

\section{Appendix C: Proof of Lemma 5.2}

Proof of Lemma 5.2 Without restriction we may assume $n<N$ (for $n=N$ the claim is trivial). Since the $\sigma$-fields $\mathcal{F}_{t}^{n}$ do not have an explicit generator, we consider filtrations which are a bit larger than $\mathbb{F}^{n}$. For each $n$ and each $m \geq n$ let $\mathbb{F}^{n, \tau_{m}}:=\left(\mathcal{F}_{t}^{n, \tau_{m}}\right)_{t \geq 0}$ where $\mathcal{F}_{t}^{n, \tau_{n}}:=\mathcal{F}_{t}^{n}$ and

$$
\mathcal{F}_{t}^{n, \tau_{m+1}}:=\left\{F \in \mathcal{F}_{\infty}^{m+1} \mid \exists F_{t} \in \mathcal{F}_{t}^{n, \tau_{m}}: F \cap\left\{\tau_{m+1}>t\right\}=F_{t} \cap\left\{\tau_{m+1}>t\right\}\right\} .
$$

(For $m=n$, these filtrations were introduced in [17], p. 81.) The filtration $\mathbb{F}^{n, \tau_{m}}$ automatically satisfies the usual conditions, and by construction $\mathcal{F}_{t}^{m} \subseteq \mathcal{F}_{t}^{n, \tau_{m}} \subseteq \mathcal{F}_{t}^{n-1, \tau_{m}}$ for every $n \leq m$. Indeed, for $n=m$, the first inclusion is trivial and the second can be found in [17], p. 81. We (may) hence continue assuming the second inclusion holds for all $n \leq m \leq M$ for some $M$. Then $\mathcal{F}_{t}^{n, \tau_{M}} \subseteq \mathcal{F}_{t}^{n-1, \tau_{M}}$ and hence 


$$
\begin{aligned}
\mathcal{F}_{t}^{n, \tau_{M+1}} & =\left\{F \in \mathcal{F}_{\infty}^{M+1} \mid \exists F_{t} \in \mathcal{F}_{t}^{n, \tau_{M}}: F \cap\left\{\tau_{M+1}>t\right\}=F_{t} \cap\left\{\tau_{M+1}>t\right\}\right\} \\
& \subseteq\left\{F \in \mathcal{F}_{\infty}^{M+1} \mid \exists F_{t} \in \mathcal{F}_{t}^{n-1, \tau_{M}}: F \cap\left\{\tau_{M+1}>t\right\}=F_{t} \cap\left\{\tau_{M+1}>t\right\}\right\} \\
& =\mathcal{F}_{t}^{n-1, \tau_{M+1}} .
\end{aligned}
$$

To prove (5.1), it is thus sufficient to show that $\mathbf{1}_{\left\{L_{t}=n\right\}} \mathbf{E}\left[X \mathbf{1}_{\left\{L_{T}=m\right\}} \mid \mathcal{F}_{t}^{n, \tau_{N}}\right]$ is equal to the right-hand side of (5.1); then the result follows by the law of iterated expectations. Let $F \in \mathcal{F}_{t}^{n, \tau_{N}}$. We first note that for $n<N$,

$$
F \cap\left\{L_{t}=n\right\}=F \cap\left\{\tau_{n+1}>t\right\} \cap \cdots \cap\left\{\tau_{N}>t\right\} \cap\left\{L_{t}=n\right\} .
$$

By definition of $\mathcal{F}_{t}^{n, \tau_{N}}, \ldots, \mathcal{F}_{t}^{n, \tau_{n+1}}$, there exist $F_{t}^{N-1} \in \mathcal{F}_{t}^{n, \tau_{N-1}}, \ldots$, $F_{t}^{n} \in \mathcal{F}_{t}^{n, \tau_{n}}=\mathcal{F}_{t}^{n}$ with $F \cap\left\{L_{t}=n\right\}=F_{t}^{N-1} \cap\left\{L_{t}=n\right\}=\cdots=F_{t}^{n} \cap\left\{L_{t}=n\right\}$. Therefore,

$$
\begin{aligned}
\mathbf{E}\left[\mathbf{1}_{F} X \mathbf{1}_{\left\{L_{t}=n\right\}} \mathbf{1}_{\left\{L_{T}=m\right\}}\right] & =\mathbf{E}\left[\mathbf{1}_{F_{t}^{n}} X \mathbf{1}_{\left\{L_{t}=n\right\}} \mathbf{1}_{\left\{L_{T}=m\right\}}\right] \\
& =\mathbf{E}\left[\mathbf{1}_{F_{t}^{n}} \mathbf{E}\left[X \mathbf{1}_{\left\{L_{t}=n\right\}} \mathbf{1}_{\left\{L_{T}=m\right\}} \mid \mathcal{F}_{t}^{n}\right]\right] \\
& =\mathbf{E}\left[\mathbf{1}_{F_{t}^{n}} \mathbf{1}_{\left\{L_{t}=n\right\}} \mathbf{E}\left[X \frac{\left.\left.\mathbf{1}_{\left\{L_{t}=n\right\}} \mathbf{1}_{\left\{L_{T}=m\right\}} \mid \mathcal{F}_{t}^{n}\right]\right]}{\mathbf{E}\left[\mathbf{1}_{\left\{L_{t}=n\right\}} \mid \mathcal{F}_{t}^{n}\right]}\right]\right. \\
& =\mathbf{E}\left[\mathbf{1}_{F} \mathbf{1}_{\left\{L_{t}=n\right\}} \frac{\mathbf{E}\left[X \mathbf{1}_{\left\{L_{t}=n\right\}} \mathbf{1}_{\left\{L_{T}=m\right\}} \mid \mathcal{F}_{t}^{n}\right]}{\mathbf{E}\left[\mathbf{1}_{\left\{L_{t}=n\right\}} \mid \mathcal{F}_{t}^{n}\right]}\right] .
\end{aligned}
$$

Equation (5.2) now follows by setting $X \equiv 1$.

\section{Appendix D: Proof of Lemma 5.6}

Proof We use the notations of Lemma 5.6, so

$$
\begin{aligned}
\widehat{P}_{n}^{\infty}(t) & :=\mathbf{E}\left[\mathbf{1}_{\left\{L_{t}=n\right\}} \mid \mathcal{F}_{\infty}^{n}\right], \\
\widehat{P}_{n, m}^{\infty}(t, T) & :=\mathbf{1}_{\left\{L_{t} \geq n\right\}} \frac{\mathbf{E}\left[\mathbf{1}_{\left\{L_{t}=n\right\}} \mathbf{1}_{\left\{L_{t}=m\right\}} \mid \mathcal{F}_{\infty}^{n}\right]}{\mathbf{E}\left[\mathbf{1}_{\left\{L_{t}=n\right\}} \mid \mathcal{F}_{\infty}^{n}\right]} .
\end{aligned}
$$

Note that $\widehat{P}_{n}^{\infty}(t)=\mathbf{1}_{\left\{L_{t} \geq n\right\}} e^{-\int_{\tau_{n}}^{t} \lambda_{s}^{n} d s}$ follows from P2 in Proposition 4.3.

Let $n$ be fixed. The proof proceeds by induction over $m$. For $m=n$, note first that $\mathbf{1}_{\left\{L_{t}=n\right\}} \mathbf{1}_{\left\{L_{T}=n\right\}}=\mathbf{1}_{\left\{L_{t} \geq n\right\}} \mathbf{1}_{\left\{\tau_{n+1}>T\right\}}$; hence with P2 it is easy to see that

$$
\widehat{P}_{n, n}^{\infty}(t, T)=\mathbf{1}_{\left\{L_{t} \geq n\right\}} e^{-\int_{t}^{T} \lambda_{s}^{n} d s} .
$$

Now the result follows from

$$
\widetilde{P}_{n, n}^{\infty}(t, T)=\frac{\mathbf{E}\left[\widehat{P}_{n}^{\infty}(t) \widehat{P}_{n, n}^{\infty}(t, T) \mid \mathcal{F}_{\infty}^{0}\right]}{\mathbf{E}\left[\widehat{P}_{n}^{\infty}(t) \mid \mathcal{F}_{\infty}^{0}\right]}=\frac{\mathbf{E}\left[\widehat{P}_{n}^{\infty}(t) e^{-\int_{t}^{T} \lambda_{s}^{n} d s} \mid \mathcal{F}_{\infty}^{0}\right]}{\mathbf{E}\left[\widehat{P}_{n}^{\infty}(t) \mid \mathcal{F}_{\infty}^{0}\right]}=e^{-\int_{t}^{T} \lambda_{s}^{n} d s}
$$


For $m>n$, we notice that $\mathbf{E}\left[\mathbf{1}_{\left\{L_{t}=n\right\}} \mathbf{1}_{\left\{L_{T} \leq m-1\right\}} \mid \mathcal{F}_{\infty}^{n}\right]$ is continuous in $T$. Indeed, it is right-continuous by the right-continuity of $L$, and left-continuous because $L_{-}$exists and

$$
\mathbf{E}\left[\mathbf{1}_{\left\{L_{t}=n\right\}}\left(\mathbf{1}_{\left\{L_{T-\leq m-1\}}\right.}-\mathbf{1}_{\left\{L_{T} \leq m-1\right\}}\right) \mathbf{1}_{F}\right] \leq \mathbf{E}\left[\mathbf{1}_{\left\{\tau_{m}=T\right\}}\right]=\mathbf{E}\left[\Delta A_{T}^{m}\right]=0
$$

for all $F \in \mathcal{F}_{\infty}^{n}$ since the predictable compensator $A^{m}$ of $\mathbf{1}_{\left\{\tau_{m} \leq t\right\}}$ is continuous a.s.

Now we let $M>n$. We may assume the result holds for $m=n, \ldots, M-1$. Then the random variable $\mathbf{1}_{\left\{L_{t}=n\right\}} \tau_{M}$ admits a right-continuous density conditional on $\mathcal{F}_{\infty}^{n}$ because the right derivative

$$
\begin{aligned}
\partial_{T} \mathbf{E}\left[\mathbf{1}_{\left\{L_{t}=n\right\}} \mathbf{1}_{\left\{\tau_{M} \leq T\right\}} \mid \mathcal{F}_{\infty}^{n}\right] & =-\partial_{T} \mathbf{E}\left[\mathbf{1}_{\left\{L_{t}=n\right\}} \mathbf{1}_{\left\{L_{T} \leq M-1\right\}} \mid \mathcal{F}_{\infty}^{n}\right] \\
& =-\widehat{P}_{n}^{\infty}(t) \sum_{m=n}^{M-1} \partial_{T} \widehat{P}_{n, m}^{\infty}(t, T) \\
& =-\widehat{P}_{n}^{\infty}(t) \sum_{m=n}^{M-1} \partial_{T} \widetilde{P}_{n, m}^{\infty}(t, T) \\
& =\widehat{P}_{n}^{\infty}(t) \widetilde{P}_{n, M-1}^{\infty}(t, T) \lambda_{T}^{M-1}
\end{aligned}
$$

exists and is right-continuous (simply apply the right derivative operator $\partial_{T}$ to $\widetilde{P}_{n, m}^{\infty}(t, T)$ for $\left.m=n, \ldots, M-1\right)$. Together with the fact that $L_{t}=n$ implies $\tau_{M}>t$, we obtain

$$
\begin{aligned}
\mathbf{E}\left[\mathbf{1}_{\left\{L_{t}=n\right\}} \mathbf{1}_{\left\{L_{T}=M\right\}} \mid \mathcal{F}_{\infty}^{n}\right] & =\mathbf{E}\left[\mathbf{1}_{\left\{L_{t}=n\right\}} \mathbf{E}\left[\mathbf{1}_{\left\{L_{T}=M\right\}} \mid \mathcal{F}_{\infty}^{M}\right] \mid \mathcal{F}_{\infty}^{n}\right] \\
& =\mathbf{E}\left[\mathbf{1}_{\left\{L_{t}=n\right\}} \mathbf{1}_{\left\{L_{T} \geq M\right\}} e^{-\int_{\tau_{M}}^{T} \lambda_{s}^{M} d s} \mid \mathcal{F}_{\infty}^{n}\right] \\
& =\mathbf{E}\left[\mathbf{1}_{\left\{L_{t}=n\right\}} \mathbf{1}_{\left\{t<\tau_{M} \leq T\right\}} e^{-\int_{\tau_{M}}^{T} \lambda_{s}^{M} d s} \mid \mathcal{F}_{\infty}^{n}\right] \\
& =\int_{t}^{T} e^{-\int_{u}^{T} \lambda_{s}^{M} d s} \partial_{u} \mathbf{E}\left[\mathbf{1}_{\left\{L_{t}=n\right\}} \mathbf{1}_{\left\{\tau_{M} \leq u\right\}} \mid \mathcal{F}_{\infty}^{n}\right] d u \\
& =\widehat{P}_{n}^{\infty}(t) \int_{t}^{T} e^{-\int_{u}^{T} \lambda_{s}^{M} d s} \widetilde{P}_{n, M-1}^{\infty}(t, u) \lambda_{u}^{M-1} d u .
\end{aligned}
$$

This implies that on the set $\left\{L_{t} \geq n\right\}, \widehat{P}_{n, M}^{\infty}(t, T)$ is equal to the $\mathcal{F}_{T}^{0}$-measurable random variable $Z_{M}:=\int_{t}^{T} e^{-\int_{u}^{T} \lambda_{s}^{M} d s} \widetilde{P}_{n, M-1}^{\infty}(t, u) \lambda_{u}^{M-1} d u$. Finally, we must show that indeed $Z_{M}=\widetilde{P}_{n, M}^{\infty}(t, T)$. By definition, we have

$$
\widetilde{P}_{n, M}^{\infty}(t, T)=\frac{\mathbf{E}\left[\widehat{P}_{n}^{\infty}(t) \widehat{P}_{n, M}^{\infty}(t, T) \mid \mathcal{F}_{\infty}^{0}\right]}{\mathbf{E}\left[\widehat{P}_{n}^{\infty}(t) \mid \mathcal{F}_{\infty}^{0}\right]}=\frac{\mathbf{E}\left[\widehat{P}_{n}^{\infty}(t) Z_{M} \mid \mathcal{F}_{\infty}^{0}\right]}{\mathbf{E}\left[\widehat{P}_{n}^{\infty}(t) \mid \mathcal{F}_{\infty}^{0}\right]}=Z_{M}
$$

Then the claim follows by induction in $M$. 


\section{References}

1. Bielecki, T.R., Rutkowski, M.: Credit Risk: Modelling, Valuation and Hedging. Springer, Berlin (2002)

2. Bielecki, T.R., Jeanblanc, M., Rutkowski, M.: Modelling and valuation of credit risk. Technical Report, Université d'Evry (2003), http://www.maths.univ-evry.fr/prepubli/189.pdf

3. Blanchet-Scalliet, C., Jeanblanc, M.: Hazard rate for credit risk and hedging defaultable contingent claims. Finance Stoch. 8, 145-159 (2004)

4. Brémaud, P., Yor, M.: Changes of filtration and of probability measures. Z. Wahrscheinlichkeitstheor. Verw. Geb. 45, 269-295 (1978)

5. Das, S.R., Duffie, D., Kapadia, N., Saita, L.: Common failings: How corporate defaults are correlated. J. Finance 62, 93-117 (2007)

6. Dellacherie, C., Meyer, P.-A.: Probabilités et Potentiel, Chapitres I-IV. Hermann, Paris (1975)

7. Dellacherie, C., Meyer, P.-A.: A propos du travail de Yor sur le grossissement des tribus. In: Séminaire de Probabilités, XII. LNM, vol. 649, pp. 70-77. Springer, Berlin (1978)

8. Dellacherie, C., Maisonneuve, B., Meyer, P.-A.: Probabilités et Potentiel, Chapitres XVII-XXIV, Processus de Markov (fin). Compléments de Calcul Stochastique. Hermann, Paris (1992)

9. Ehlers, P.: Pricing Credit Derivatives. Ph.D. Thesis 17274, ETH Zurich (2007)

10. Ehlers, P., Schönbucher, P.J.: Dynamic credit portfolio derivatives pricing. Working Paper, ETH Zurich, July 2006, http://www.defaultrisk.com/pp_price_76.htm

11. Elliott, R.J., Jeanblanc, M., Yor, M.: On models of default risk. Math. Finance 10, 179-196 (2000)

12. Errais, E., Giesecke, K., Goldberg, L.R.: Pricing credit from the top down with affine point processes. Working Paper, June 2006, http://www.barra.com/products/pdfs/pricing_credit.pdf

13. Frey, R., Backhaus, J.: Portfolio credit risk models with interacting default intensities: A Markovian approach. Working Paper, Department of Mathematics, Universität Leipzig (2004), http://www.math.uni-leipzig.de/ frey/publications-frey.html

14. Giesecke, K., Goldberg, L.: A top down approach to multi-name credit. Working Paper, Cornell University, September 2005, http://www.stanford.edu/dept/MSandE/people/faculty/ giesecke/topdown.pdf

15. Heath, D., Jarrow, R., Morton, A.: Bond pricing and the term structure of interest rates: A new methodology for contingent claims valuation. Econometrica 60, 77-105 (1992)

16. Jeanblanc, M., Rutkowski, M.: Modelling of default risk: Mathematical tools. Working Paper, March 2000 (contact http://www.maths.univ-evry.fr/pages_perso/jeanblanc)

17. Jeulin, T., Yor, M.: Grossissement d'une filtration et semi-martingales: Formules explicites. In: Séminaire de Probabilités, XII. LNM, vol. 649, pp. 78-97. Springer, Berlin (1978)

18. Kusuoka, S.: A remark on default risk models. Adv. Math. Econ. 1, 69-82 (1999)

19. Lando, D.: On Cox processes and credit risky securities. Rev. Deriv. Res. 2, 99-120 (1998)

20. Longstaff, F.A., Schwartz, E.S.: Valuing American options by simulation: A simple least-squares approach. Rev. Financ. Stud. 14, 113-147 (2001)

21. Protter, P.: Stochastic Integration and Differential Equations, 2nd edn. Springer, Berlin (2004)

22. Schönbucher, P.J.: Portfolio losses and the term structure of loss transition rates: A new methodology for the pricing of portfolio credit derivatives. Working Paper, ETH Zurich, July 2006, http://www.defaultrisk.com/pp_model_74.htm

23. Sidenius, J., Piterbarg, V., Andersen, L.: A new framework for dynamic credit portfolio loss modelling. Working Paper, December 2004, http://www.defaultrisk.com/pp_model_83.htm

24. Sidenius, J., Piterbarg, V., Andersen, L.: A new framework for dynamic credit portfolio loss modeling. Int. J. Theor. Appl. Finance 11, 163-197 (2008)

25. Wheeden, R.L., Zygmund, A.: Measure and Integral. Dekker, New York (1977) 